ARTICLE

Received 27 Aug 2014 | Accepted 3 Mar 2015 | Published 16 Apr $2015 \quad$ DOl: 10.1038/ncomms7842

\title{
Rheb activation disrupts spine synapse formation through accumulation of syntenin in tuberous sclerosis complex
}

Hiroko Sugiura ${ }^{1, \star}$, Shin Yasuda ${ }^{1, \star}$, Shutaro Katsurabayashi ${ }^{2, \star}$, Hiroyuki Kawano ${ }^{2}$, Kentaro Endo $^{3}$, Kotaro Takasaki ${ }^{2}$, Katsunori Iwasaki ${ }^{2}$, Masumi Ichikawa ${ }^{3}$, Toshiyuki Kobayashi ${ }^{4}, \mathrm{Okio} \mathrm{Hino}^{4}$ \& Kanato Yamagata ${ }^{1}$

Rheb is a small GTP-binding protein and its GTPase activity is activated by the complex of Tsc1 and Tsc2 whose mutations cause tuberous sclerosis complex (TSC). We previously reported that cultured TSC neurons showed impaired spine synapse morphogenesis in an mTORC1-independent manner. Here we show that the PDZ protein syntenin preferentially binds to the GDP-bound form of Rheb. The levels of syntenin are significantly higher in TSC neurons than in wild-type neurons because the Rheb-GDP-syntenin complex is prone to proteasomal degradation. Accumulated syntenin in TSC neurons disrupts spine synapse formation through inhibition of the association between syndecan-2 and calcium/calmodulindependent serine protein kinase. Instead, syntenin enhances excitatory shaft synapse formation on dendrites by interacting with ephrinB3. Downregulation of syntenin in TSC neurons restores both spine and shaft synapse densities. These findings suggest that Rheb-syntenin signalling may be a novel therapeutic target for abnormalities in spine and shaft synapses in TSC neurons.

\footnotetext{
${ }^{1}$ Neural Plasticity Project, Tokyo Metropolitan Institute of Medical Science, Tokyo 156-8506, Japan. ${ }^{2}$ Department of Neuropharmacology, Faculty of Pharmaceutical Sciences, Fukuoka University, Fukuoka 814-0180, Japan. ${ }^{3}$ Center of Basic Technology Research, Tokyo Metropolitan Institute of Medical Science, Tokyo 156-8506, Japan. ${ }^{4}$ Department of Pathology and Oncology, Juntendo University, School of Medicine, Tokyo 113-8421, Japan. * These authors contributed equally to this work. Correspondence and requests for materials should be addressed to K.Y. (email: yamagata-kn@igakuken.or.jp).
} 
$\mathrm{D}$ endritic spine formation and synaptogenesis are often preceded by a period in which numerous elongated filopodia extend from developing dendrites. The impairment in the conversion of filopodia to dendritic spines may lead to childhood cognitive disorders ${ }^{1}$. Synaptic abnormalities in neurodevelopmental disorders may be caused by mutations that affect synaptic proteins. For example, mutations in fmrl, identified in fragile $\mathrm{X}$ syndrome, alter the properties of the FMRP protein, resulting in spine dysmorphogenesis ${ }^{2}$. The E3 ubiquitin ligase Ube3A, linked to Angelman syndrome, also controls spine formation ${ }^{3}$. Moreover, another autism-related protein neurobeachin regulates spine density and shaft synapse formation ${ }^{4}$. Thus, childhood mental disorders may be accompanied by morphological abnormalities of excitatory spine and/or shaft synapses.

Tuberous sclerosis complex (TSC) is a systemic genetic disease that causes the growth of hamartomas in the brain and other organs $s^{5}$. The neuropsychiatric symptoms of TSC patients include intellectual disability, epilepsy and/or autism ${ }^{5}$. TSC results from inactivating mutations in either Tsc1 (ref. 6) or Tsc2 (ref. 7), which encode hamartin and tuberin, respectively. Hamartin and tuberin form a complex that exhibits guanosine triphosphatase (GTPase)-activating protein activity towards the small GTPbinding protein $\mathrm{Rheb}^{8-10}$. Inactivating mutations in $T s c 1$ or $T s c 2$ result in the accumulation of the GTP-bound form of Rheb, which activates mammalian target of rapamycin complex 1 (mTORC1; ref. 11). A Golgi study of surgical specimens from a TSC patient reported that cortical neurons showed aberrant spine development ${ }^{12}$. Several lines of evidence also suggest that $T s c 1^{+/-}$or $T s c 2^{+/-}$rodents show impairments in dendritic spine formation ${ }^{13,14}$ and cognitive behaviours ${ }^{15}$, suggesting that the activation of mTORC1 in TSC neurons may cause memory disturbances, likely through dendritic spine abnormalities. However, the mTORC1 signalling pathway has been shown to be activated during learning and is required for spatial memory formation. This discrepancy may indicate that an mTORC1-independent mechanism may be involved in memory impairments in TSC.

Syndecan-2 is a transmembrane heparan sulphate proteoglycan that promotes spine formation ${ }^{16-18}$. The C-terminal EFYA motif of syndecan-2 is necessary for dendritic spinogenesis, and this motif binds to the PSD-95, Dlg and ZO1 (PDZ) domains of calcium/calmodulin-dependent serine protein kinase (CASK). CASK is known to associate with F-actin via its binding to protein 4.1 (ref. 18), and it may mediate spine morphogenesis through syndecan-2. However, the $\mathrm{C}$ terminus of syndecan-2 also binds to syntenin, a scaffold protein with two PDZ domains ${ }^{19}$. In contrast to CASK, overexpression of syntenin in hippocampal neurons increases filopodia-like dendritic protrusions ${ }^{20}$, suggesting that syntenin competes with CASK for syndecan-2.

In the present study, we propose a novel mechanism for dendritic spine morphogenesis. We previously showed that activation of Rheb, but not mTORC1, impaired spine synapse formation in TSC neurons ${ }^{14}$. To further elucidate this mechanism, we sought to detect a new Rheb-mediated signalling pathway in the brain and to identify syntenin as a novel Rheb-binding protein. Syntenin preferentially binds to the GDP form of Rheb, and this complex is prone to proteasomal degradation in wild-type (WT) neurons. The consequent reduction in syntenin levels allows CASK to associate with syndecan-2, leading to stabilization of spine structure. In $T s c 2^{+1-}$ neurons, the syntenin released from Rheb-GTP associates with syndecan-2, resulting in decreased spine synapse density. Syntenin also interacts with ephrinB3 and increases the density of excitatory shaft synapses. In fact, downregulation of syntenin restores spine and shaft synapse densities in $T s c 2^{+1-}$ neurons. These results suggest that syntenin is a regulator of spine and shaft synapse density, and link syntenin to a novel pathophysiological mechanism implicated in neurodevelopmental disorders.

\section{Results}

Rheb associates with syntenin. We previously reported that dendritic spine synapse density was decreased in TSC neurons through an mTOR-independent mechanism ${ }^{14}$. We found that activation of Rheb, but not mTORC1, reduced spine synapse density, and the expression of a dominant-negative form of Rheb or treatment with farnesyl transferase inhibitors largely restored spine synapse density in $T s c 2^{+/-}$neurons. These results suggest that the mTOR-independent mechanism controlling spine synapse density involves Rheb, and that this mechanism may be dysregulated in TSC neurons.

To elucidate this mechanism, in this study, we searched for a Rheb-associating protein using the yeast two-hybrid system. We identified a PDZ domain-containing scaffold protein, syntenin ${ }^{19}$, as a novel Rheb-binding partner. The potential interaction between Rheb and syntenin was confirmed by coimmunoprecipitation of Rheb with syntenin from rat brain lysates (Fig. 1a). Furthermore, Rheb ${ }^{\text {D60I }}$ protein was pulled down with glutathione S-transferase (GST)-fused syntenin (Fig. 1b). The association of syntenin with Rheb appeared to depend on the nucleotide-binding state of Rheb, as the GDP-bound form (Rheb ${ }^{\mathrm{D} 60 \mathrm{I}}$ ) exhibited a significantly stronger binding to syntenin than did the GTP-bound form (Rheb ${ }^{864 V}$; Fig. 1c,d).

We localized the Rheb-binding region of syntenin to its C-terminal segment but not to either PDZ domain because the affinity of Rheb to syntenin was significantly reduced by the C-terminal deletions $(\Delta 278-300$ and $\Delta 289-300)$ but not by the mPDZ1 or mPDZ2 (ref. 19; Fig. 1e,f). Furthermore, syntenin $^{\Delta C(289-294)}$ showed no interaction with Rheb (Fig. 1g), indicating that the Rheb-binding region of syntenin is localized between residues 289 and 294. Conversely, syntenin interacted with the switch I region (also called the effector region) of Rheb in pull-down experiments. Specifically, a T38M, but not I39K, mutation in Rheb ${ }^{\mathrm{D} 60 \mathrm{I}}$ reduced the binding to syntenin (Fig. 1h), suggesting that residues exposed only in Rheb-GDP form may mediate the binding of the two proteins ${ }^{21}$.

Syntenin regulates dendritic spine synapse density. To elucidate the role of the Rheb-syntenin interaction in spine synapse morphogenesis, we first compared syntenin levels in WT and TSC neurons. We used spontaneous Tsc2-mutated rats (Eker) as a model of $\mathrm{TSC}^{22}$. We cultured hippocampal neurons of WT and heterozygous Eker $\left(T s c 2^{+/-}\right)$rat embryos, fixed the cultures at 21 days in vitro (DIV) and immunostained the neurons with antisyntenin antibody. As shown in Fig. 2a,b, syntenin levels were significantly higher in $T s c 2^{+/-}$neurons than in WT neurons. This result was consistent with an increase in syntenin levels in $T s c 2^{+1-}$ brains compared with those in WT brains (Fig. $2 \mathrm{c}, \mathrm{d}$ ), suggesting that the Tsc2 mutation may affect neuronal syntenin levels, possibly via Rheb.

To test whether the nucleotide-binding state of Rheb regulates syntenin levels, we overexpressed the GTP-bound form of Rheb $\left(\mathrm{Rheb}^{\mathrm{Q} 64 \mathrm{~V}}\right.$ ) in cultured hippocampal neurons ${ }^{14}$. The expression of active Rheb significantly increased both the somatic and dendritic levels of syntenin in hippocampal neurons compared with adjacent untransfected neurons (Supplementary Fig. 1a,b). By contrast, the expression of Rheb ${ }^{\text {D60I }}$ decreased syntenin levels in cultured neurons (Supplementary Fig. 1c,d). These results suggest that the nucleotide-binding state of Rheb controls the syntenin levels in hippocampal neurons. 
To confirm the effects of neuronal syntenin levels on spine and synapse formation, we overexpressed or knocked down syntenin in primary cultures of hippocampal neurons. Overexpression of syntenin in WT neurons decreased and increased dendritic protrusion width and length, respectively (Fig. 2e,f). Furthermore, syntenin expression drastically reduced spine synapse density (Fig. 2e,g), as observed previously in $\mathrm{Rheb}^{\mathrm{Q} 64 \mathrm{~V}_{\text {-expressing } \mathrm{WT}}}$ neurons or $T s c 2^{+I-}$ neurons ${ }^{14}$. We then knocked down the endogenous syntenin levels in $T s c 2^{+/-}$neurons, whose syntenin levels were higher than those in WT neurons. Syntenin short interfering RNA (siRNA) reduced the expression of exogenous syntenin by $92.4 \%$ in HEK293T cells (Supplementary Fig. 1e). The transfection of syntenin siRNA into $T s c 2^{+1-}$ neurons reduced the endogenous syntenin levels (Supplementary Fig. 1f) and induced wider and shorter dendritic protrusions (Fig. 2h,i). Syntenin knockdown also drastically enhanced spine synapse density in $T s c 2^{+/-}$neurons (Fig. 2j). Accordingly, a reduction in syntenin levels may restore spine synapse density in $T s c 2^{+1-}$ neurons. By contrast, unexpectedly, knockdown of syntenin in WT neurons also disrupted spine synapse morphogenesis (Fig. 2h,j) and produced narrower and longer filopodia-like protrusions (Fig. 2i). Thus, both increasing and decreasing syntenin levels can affect spine synapse formation, suggesting that neuronal syntenin levels must be maintained within a narrow range for normal spine development.

The Rheb-syntenin complex is degraded by proteasomes. What mechanism underlies the increase in syntenin levels in TSC neurons? One possibility is the upregulation of syntenin production, and another is the inhibition of syntenin degradation. The former is unlikely because the $\mathrm{mTORC} 1$ inhibitor rapamycin failed to reduce syntenin levels in $T s c 2^{+1-}$ neurons (unpublished observations). Recent work has demonstrated that the proteasome system regulates activity-dependent spine outgrowth $^{23}$. Therefore, we assumed that syntenin levels may be regulated by the ubiquitin-proteasome system and that normal steady-state levels of syntenin degradation are required for spine synapse development. We treated Rheb ${ }^{\text {D60I }}$-expressing Tsc2 ${ }^{+1-}$ neurons with the proteasomal inhibitors lactacystin $(0.5 \mu \mathrm{M})$ or MG132 $(0.1 \mu \mathrm{M})$ for 2 days, and observed that the Rheb ${ }^{\mathrm{D} 60 \mathrm{I}}$ induced decreases and increases in syntenin levels and spine synapse density, respectively, were prevented by either inhibitor (Fig. 3a-d; unpublished data). Proteasomal inhibitor alone did not change spine synapse density in WT neurons (Fig. 3d), consistent with a previous study ${ }^{23}$. Furthermore, knockdown of
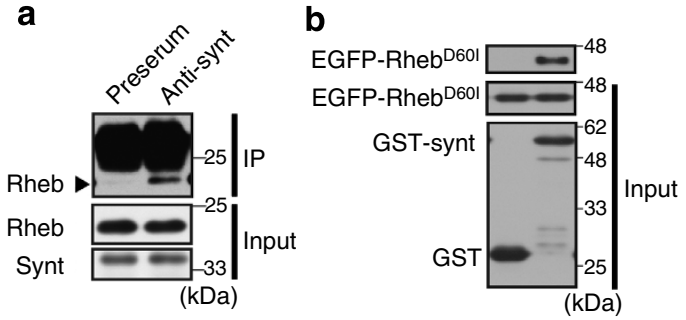

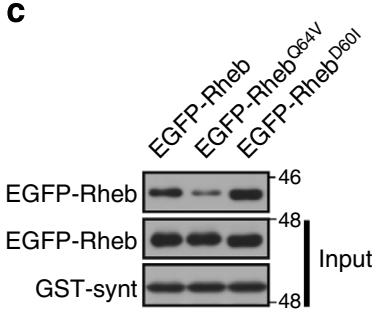

(kDa)
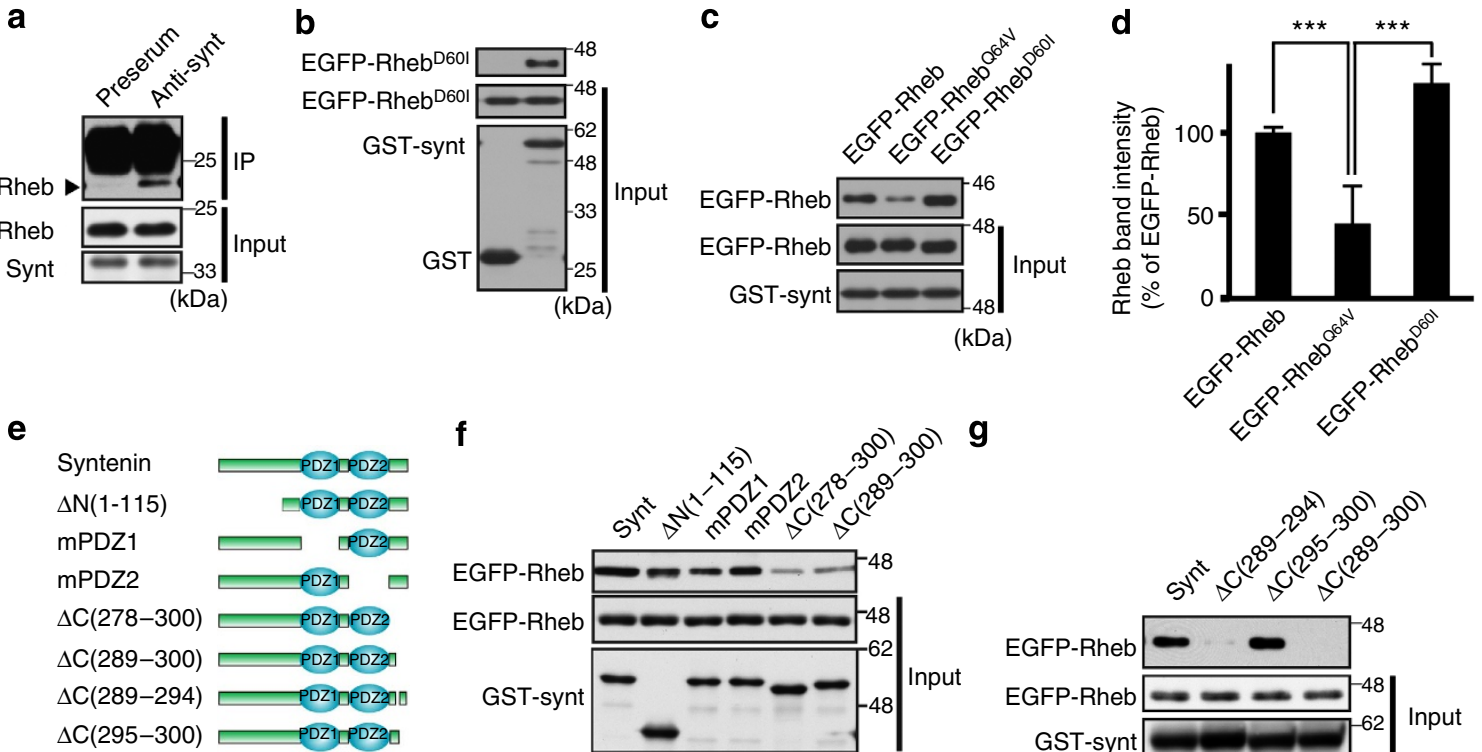

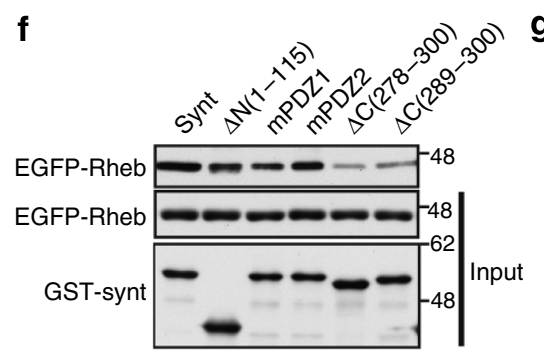

$(\mathrm{kDa})$

g

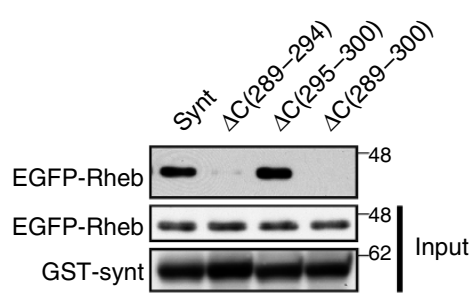

$(\mathrm{kDa})$

$\mathbf{h}$

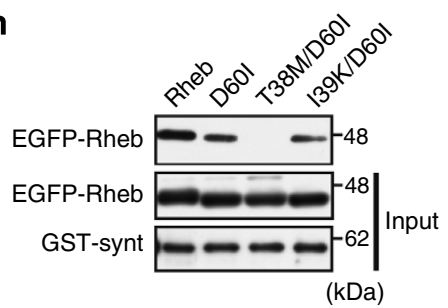

Figure 1 | Rheb associates with syntenin. (a) Rat brain lysates were immunoprecipitated (IP) with preimmune serum (preserum, lane 1) or anti-syntenin (synt) antibody (lane 2). Rheb in the precipitates was detected using immunoblotting. (b) Pull-down of Rheb ${ }^{\text {D60I }}$ with GST-syntenin, not with GST alone,

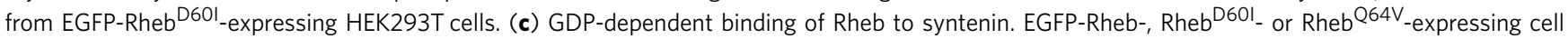
lysates were precipitated with GST-syntenin. The precipitates were immunoblotted with anti-GFP antibody. (d) Quantification of EGFP-Rheb pulled down with GST-syntenin as shown in $\mathbf{c}\left(n=8\right.$ independent experiments, ${ }^{\star \star \star} P<0.001$, unpaired, two-tailed $t$-test). (e) Schematic diagram of WT syntenin and various syntenin mutants: N-terminal domain-deleted ( $\triangle N(1-115)$ ), PDZ1 domain-mutated (mPDZ1), PDZ2 domain-mutated (mPDZ2) and C-terminal domain-deleted ( $\triangle C(278-300), \Delta C(289-300), \Delta C(289-294)$ and $\triangle C(295-300))$. (f,g) Identification of the Rheb-binding domain of syntenin. Each syntenin mutant fused to GST was examined for binding to Rheb ${ }^{\text {D60l }}$ in HEK293T cells using pull-down assays. (h) Rheb ${ }^{\text {D60l }}$, Rheb ${ }^{\text {T38M/D60l }}$ or Rheb ${ }^{139 K / D 60 l}$ mutants expressed in HEK293T cells were examined for binding to GST-syntenin using pull-down assays. Blots in a-c,f-h) are cropped and full-length blots are presented in Supplementary Fig. 6a-f. All data are presented as mean \pm s.e.m. 
a

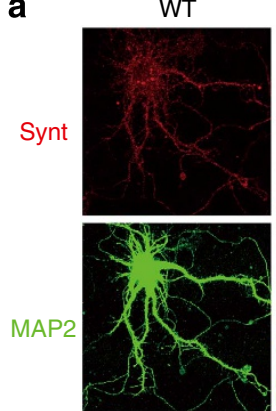

e

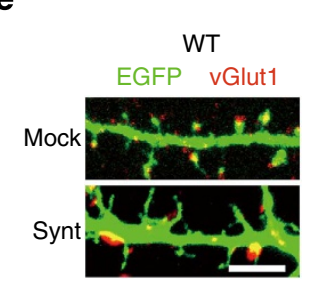

$\mathrm{Tsc}^{+/-}$

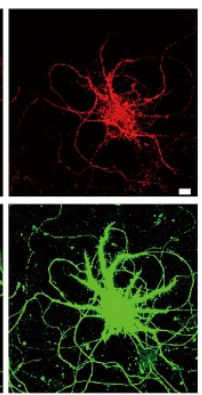

b

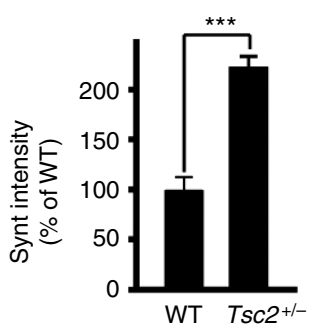

c

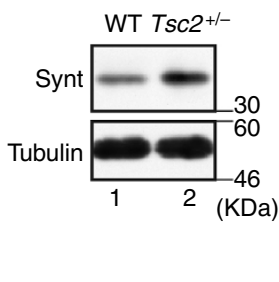

d

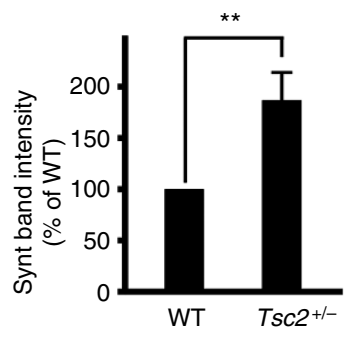

f

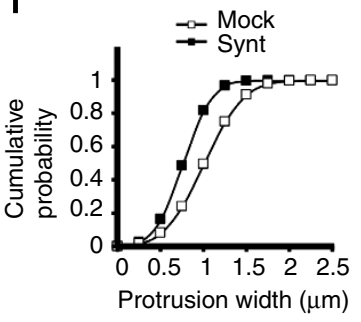

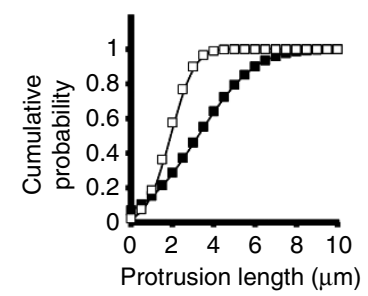

g

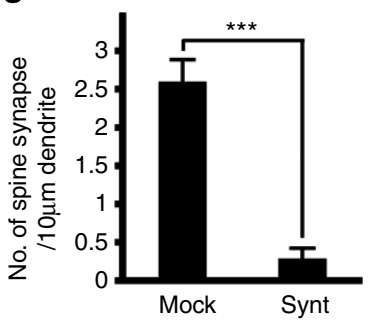

h

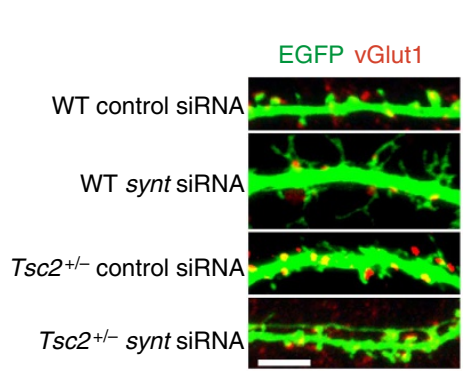

j

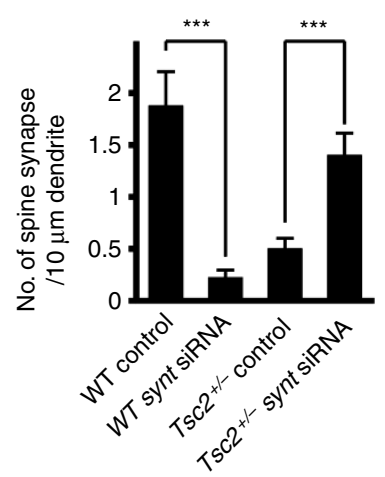

i $\quad \square-W T$ control siRNA $=-$ WT synt siRNA $\underline{-1}$ Tsc2 $^{+/-}$synt siRNA
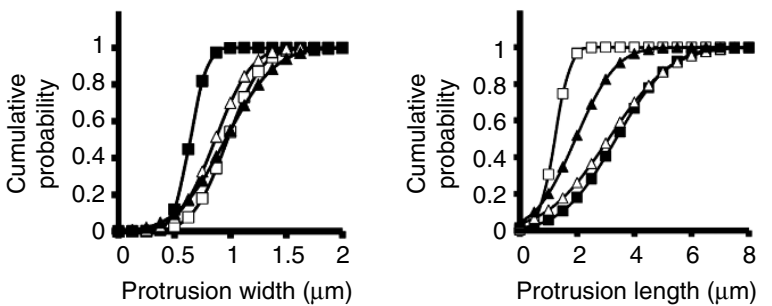

Figure 2 | Syntenin negatively regulates spine synapse formation. (a) Immunostaining of WT (left) and Tsc2 ${ }^{+/-}$(right) neurons using anti-syntenin (anti-synt; top) and MAP2 (bottom) antibodies. Scale bar, $10 \mu \mathrm{m}$. (b) Quantification of the syntenin immunoreactivity as shown in a, normalized to that of MAP2 ( $n=5 / 3$ (WT), 6/3 (Tsc2 ${ }^{+/-}$) (DIV 16) neurons/experiments; ${ }^{* \star *} P<0.001$, unpaired, two-tailed $t$-test). (c) Levels of syntenin and tubulin in WT (lane 1) and Tsc2 ${ }^{+/-}$rat brains (lane 2). These blots are cropped and full-length blots are presented in Supplementary Fig. 6g. (d) Quantification of syntenin levels in Tsc2 ${ }^{+1}$ - rat brains normalized to WT levels ( $n=8$ independent experiments, ${ }^{\star \star} P<0.01$, unpaired, two-tailed $t$-test). (e) Dendrites of EGFP- and mock- (upper) or ECFP-syntenin (synt)-expressing (lower) WT neurons at DIV 21. The neurons were immunolabelled with anti-vGlut1. Scale bar, $10 \mu \mathrm{m}$. (f) Cumulative probability distributions of the protrusion width and length as shown in $\mathbf{e}$. The syntenin-expressing neurons demonstrate narrower $(D=0.3596, P<0.001)$ and longer $(D=0.3623, P<0.001)$ dendritic protrusions than mock transfectants (Kolmogorov-Smirnov test). (g) Quantification of vGlut1-positive spine synapse density in the neurons transfected as in e. In total, 496/5/3 (mock) and 568/6/3 (syntenin) protrusions/neurons/ experiments, respectively, were analysed ( ${ }^{\star \star} P<0.001$, two-tailed $t$-test). (h) Dendrites of WT and Tsc2 ${ }^{+/-}$neurons transfected with syntenin siRNA or scrambled siRNA. Scale bar, $10 \mu \mathrm{m}$. (i) Cumulative probability plots of the protrusion width and length as shown in $\mathbf{h}$. Syntenin siRNA-expressing Tsc2 ${ }^{+/-}$ neurons show wider $(D=0.1253, P<0.001)$ and shorter $(D=0.3474, P<0.001)$ dendritic protrusions than scrambled siRNA (Kolmogorov-Smirnov test). (j) Quantitative analysis of the spine synapse densities as shown in $\mathbf{h}(n=500 / 5 / 3$ (WT, syntenin siRNA), 500/5/3 (WT, scrambled siRNA), 1,034/10/5 $\left(T s c 2^{+/-}\right.$, syntenin siRNA) and 1,146/10/5 (Tsc2 ${ }^{+/-}$, scrambled siRNA) protrusions/neurons/experiments, ${ }^{\star \star \star} P<0.001$, two-way analysis of variance, (ANOVA) post hoc Tukey test). All data are presented as mean \pm s.e.m. 
syntenin rescued spine synapse density in lactacystin-treated neurons (Fig. 3c,d). Thus, Rheb ${ }^{\text {D6I }}$-induced spine synapse morphogenesis in $T s c 2^{+1-}$ neurons may be closely related to the reduction in syntenin levels by proteasomal degradation.

To further elucidate the mechanism of syntenin downregulation, we investigated whether proteasomal degradation of syntenin depends on the nucleotide-binding state of Rheb. Syntenin was co-expressed with Rheb ${ }^{\mathrm{D} 60 \mathrm{I}}$ or Rheb ${ }^{\mathrm{Q} 64 \mathrm{~V}}$ in COS-7 cells, which were treated with MG132 (ref. 24) or untreated. We compared the syntenin levels between MG132-treated and -untreated cells, and observed that the proteasomal degradation of syntenin was enhanced in the Rheb-GDP state compared with that in the Rheb-GTP state (Fig. 3e,f). Moreover, the GDP-bound form of Rheb was more sensitive to proteasomal degradation than Rheb-GTP (Fig. 3e,g). Next, we tested whether the association between syntenin and Rheb is required for the proteasomal degradation of both proteins. We co-expressed Rheb ${ }^{\mathrm{D} 60 \mathrm{I}}$ with either intact syntenin or syntenin $\Delta C(289-294)$, a mutant with a deletion of the Rheb-binding site, in COS-7 cells. Syntenin $\Delta \mathrm{C}(289-294)$ drastically reduced the degradation of Rheb $^{\text {D60I }}$ (Fig. 3h,i), indicating that the proteasomal degradation of Rheb is enhanced by its interaction with syntenin.

Syntenin competes with CASK for association with syndecan-2. To investigate the mechanism underlying the syntenin-induced decrease in spine synapse density, we identified the domain of syntenin that participates in this process. We created siRNAresistant forms of N-terminal-deleted (syntenin $\left.{ }^{\star \Delta N(1-115)}\right)$, PDZ1mutated (syntenin $\left.{ }^{\star m P D Z 1}\right), \mathrm{PDZ2}$-mutated $\left(\right.$ syntenin $\left.^{\star m P D Z 2}\right)$ and C-terminal-deleted (syntenin ${ }^{\star \Delta C(289-300)}$ ) syntenin expression constructs. We co-transfected each syntenin mutant with syntenin siRNA to downregulate endogenous syntenin in primary neurons. Among the various syntenin mutants, only synte$n i n^{* m P D Z 2}$ reversed the spine synapse density (Supplementary Fig. $2 \mathrm{a}-\mathrm{c}$ ), suggesting that the PDZ2 domain of syntenin mediates spine synapse dysmorphogenesis.

The PDZ2 domain of syntenin has been associated with various proteins ${ }^{25}$. Of the more than 20 syntenin-binding proteins, only syndecan- 2 has been demonstrated to regulate dendritic spine formation ${ }^{17,18,26}$. To clarify whether syndecan-2 is involved in aberrant spinogenesis in TSC neurons, we downregulated both syntenin and syndecan-2 in $T s c 2^{+1-}$ neurons. Syndecan-2 siRNA reduced endogenous and exogenous syndecan-2 levels in cultured neurons and HEK293T cells, respectively (Supplementary Fig. 2d,e). Knockdown of syndecan-2 alone abolished normal spine synapse formation in WT neurons, confirming that syndecan-2 plays a crucial role in normal spine development. Similarly, syndecan-2 knockdown in $T s c 2^{+/}-$neurons did not restore spine formation, but concomitant reduction in syndecan-2 abolished the recovery of spine synapse density produced by syntenin siRNA (Fig. 4a,b). These results suggest that increased syntenin may disrupt spine synapse formation through association with syndecan-2.

Similar to syntenin, CASK interacts with the C-terminal EFYA motif of syndecan-2 and promotes dendritic spine maturation ${ }^{16,27}$. We examined whether the binding of syndecan-2 to CASK and syntenin is mutually exclusive. We first tested the ability of CASK to induce spine synapse formation. The overexpression of CASK in Tsc2 ${ }^{+1}$ neurons also increased spine synapse density as well as syntenin knockdown (Fig. 4c,d); however, syntenin levels were not changed by CASK expression (Supplementary Fig. 2f,g), suggesting that an increase in CASK relative to syntenin restores spine synapse formation in $T s c 2^{+1-}$ neurons. Further increases in syntenin impaired the ability of CASK to enhance spine synapse formation (Fig. 4e,f). We then examined whether the inhibitory effects of syntenin reflect competition between CASK and syntenin for the binding to the $\mathrm{C}$ terminus of syndecan-2. We fused GST to the intracellular domain (IC) of syndecan-2 to pull-down CASK protein. In the absence of FLAG-syntenin, CASK was pulled down with GST-syndecan-2 IC. However, increasing the amount of FLAGsyntenin in cell lysates suppressed the pull-down of CASK with GST-syndecan-2 in a dose-dependent manner (Fig. 4g,h), indicating that syntenin competes with CASK for the binding to syndecan- 2 .

The protein 4.1-binding motif of CASK is involved in spine morphogenesis ${ }^{27}$. Protein 4.1 binds to spectrin ${ }^{28}$ and promotes the interaction between spectrin and filamentous actin ${ }^{28,29}$; thus, CASK may act as a linker between transmembrane proteins and the actin cytoskeleton, thereby stabilizing dendritic spines. We therefore sought to determine whether the protein 4.1-binding site of CASK plays a pivotal role in spine synapse morphogenesis. The CASK mutant lacking the protein 4.1-binding motif

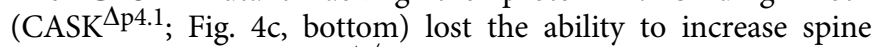
synapse density in $T s c 2^{+/-}$neurons (Fig. 4d). These results indicate that the protein 4.1-binding site of CASK is required for spine synapse formation in $T s c 2^{+/-}$neurons. Taken together, these findings indicate that syntenin may reduce spine synapse density by competing with CASK and preventing it from stabilizing spine morphology via protein 4.1 .

Binding of syntenin to ephrinB3 increases shaft synapses. We previously showed that in $T s c 2^{+/-}$neurons, excitatory synapses were rarely located on dendritic protrusions but preferentially localized to dendritic shafts ${ }^{14}$. Syntenin has been demonstrated to be a downstream effector of ephrinB3 (ref. 30), which modulates glutamatergic synaptic density ${ }^{31}$, particularly on shaft synapses ${ }^{32}$. To test whether syntenin is involved in excitatory shaft synapse formation, we knocked down syntenin in WT and Tsc2 ${ }^{+/-}$ neurons. Downregulation of syntenin drastically reduced excitatory shaft synapse density in $T s c 2^{+/-}$neurons but not in WT neurons (Fig. 5a). Next, we examined the involvement of ephrinB3 in the syntenin-mediated shaft synapse formation. We knocked down the endogenous ephrinB3 in Tsc2 $2^{+1-}$ hippocampal neurons using ephrinB3 siRNA (Supplementary Fig. 3a,b). Eleven days after the ephrinB3 siRNA transfection, we observed a significant reduction in vGlutl puncta on the dendritic shafts in Tsc2 $+1-$ neurons but not in WT neurons (Fig. 5b). EphrinB3 knockdown did not affect spine synapse density in either WT or $T s c 2^{+/-}$neurons (Fig. 5b). Conversely, overexpression of ephrinB3 increased excitatory shaft synapse density in WT neurons (Fig. 5c), and simultaneous expression of syntenin siRNA effectively inhibited this increase (Fig. 5c), suggesting that ephrinB3 may regulate shaft synapse density via syntenin. In our experiment, the overexpression of ephrinB3 significantly reduced spine synapse density, which was inconsistent with a previous study ${ }^{32}$. The reason for this difference is unclear, but our overexpression of ephrinB3 plasmid was longer and stronger (10 days under the control of the CAG promoter) than that in the other study ${ }^{32}$ (4 days under the control of the cytomegalovirus promoter). Because ephrinB3 regulates synapse density by inhibiting the postsynaptic mitogenactivated protein kinase signalling ${ }^{31}$ that is required for increases in spine density ${ }^{33}$, long and strong expression of ephrinB3 might reduce spine synapse density. Future studies will be needed to characterize this mechanism.

To examine the direct interaction between ephrinB3 and syntenin, we co-immunoprecipitated ephrinB3 with syntenin from HEK293T cells expressing green fluorescent protein (GFP)syntenin and ephrinB3 (Fig. 5d). This binding likely occurred 
through a PDZ domain because the ephrinB3 mutant lacking the PDZ recognition motif was not associated with syntenin (Fig. 5e). Together, these results indicate that syntenin may enhance shaft synapse formation by binding to ephrinB3 through the PDZ domain.

Excitatory shaft synapses in $\mathbf{T s c 2}{ }^{+/-}$neurons are functional. In a previous work, to investigate excitatory synaptic transmission in TSC neurons, we systematically investigated the electrophysiological properties of $\mathrm{WT}$ and $T s c 2^{+/-}$neurons ${ }^{14}$. Unexpectedly, Tsc2 $2^{+/-}$neurons showed sufficient synaptic transmission, with no significant differences in presynaptic or postsynaptic features between $\mathrm{WT}$ and $T s c 2^{+/-}$neurons. Therefore, we proposed that increased shaft synapses in $T s c 2^{+1-}$ neurons may functionally compensate for the excitatory synaptic transmission of decreased spine synapses. To test this hypothesis, we reduced shaft synapse density in

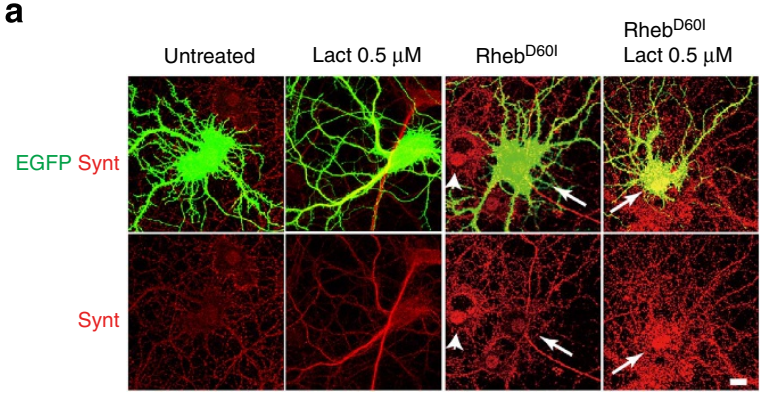

a

C

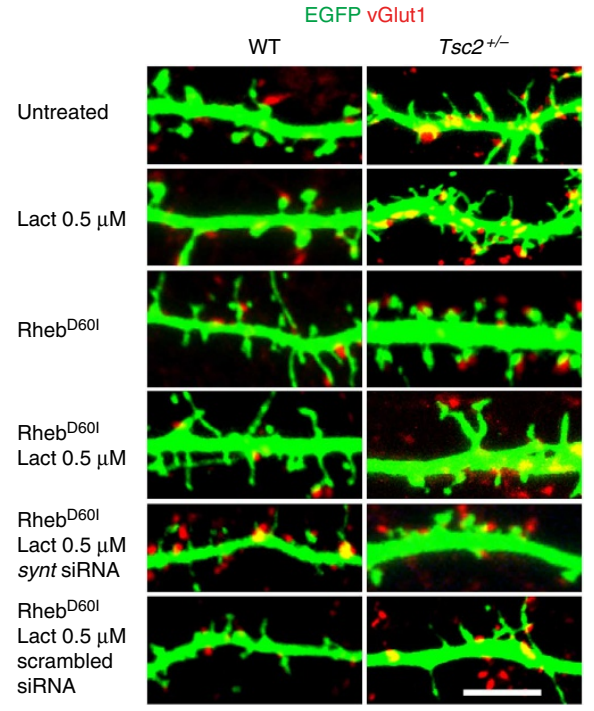

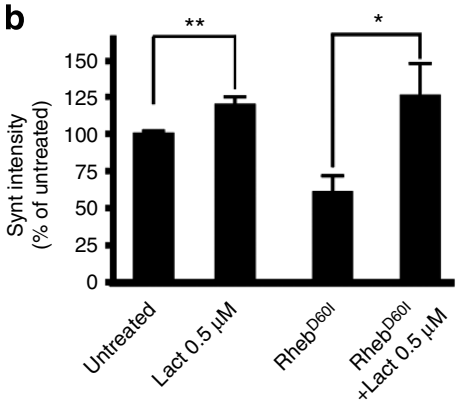

d

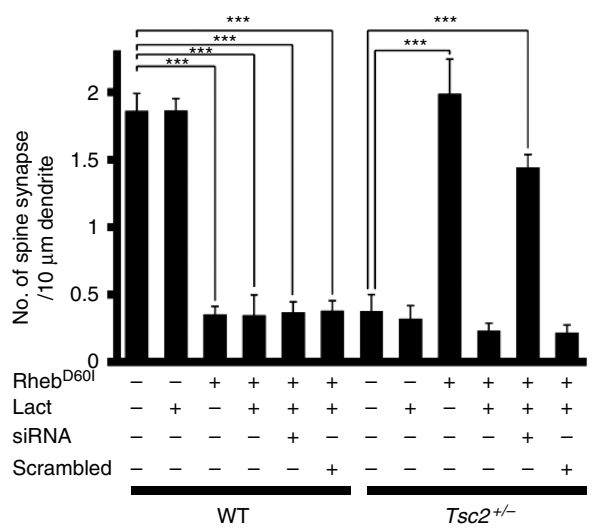

$\mathbf{e}$

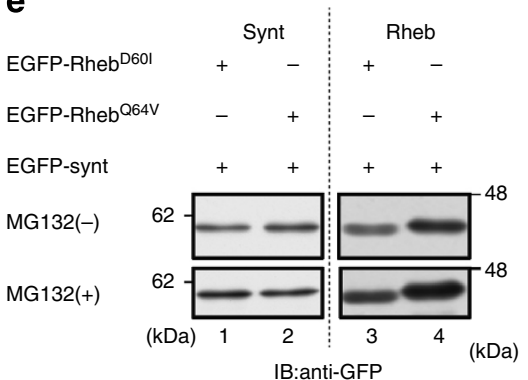

f

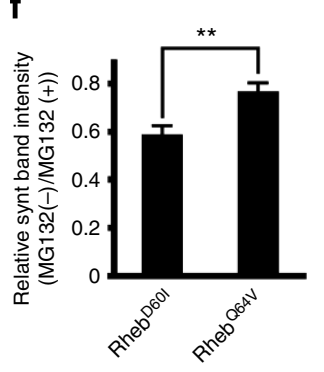

g

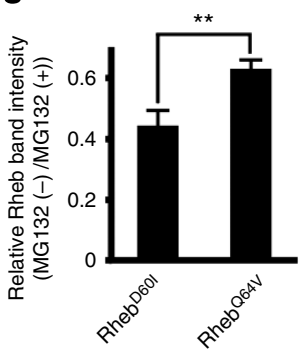

h

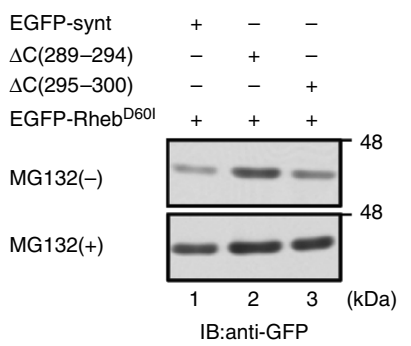

i

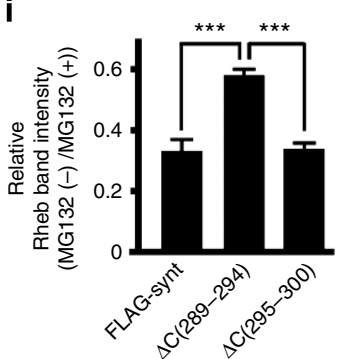


autaptic WT and $T s c 2^{+/-}$neurons by expressing ephrinB3 siRNA, and we electrophysiologically recorded synaptic transmission in these neurons (Fig. 6a-g). Although the control siRNA did not affect synaptic transmission in either WT or $\mathrm{Tsc}^{+/-}$neurons (Supplementary Fig. 4a-g), ephrinB3 siRNA markedly reduced the excitatory postsynaptic current (EPSC) amplitude and miniature EPSC (mEPSC) frequency without changes in the probability of vesicular release in $\mathrm{Tsc}^{+/-}$ neurons (Fig. 6a-f). These data suggest that excitatory synaptic transmission between $T s c 2^{+1-}$ neurons is functionally compensated for by increases in the ephrinB3-mediated shaft synapses, leading to the apparent stabilization of synaptic transmission in $T s c 2^{+/-}$neurons. Furthermore, we immunostained $T s c 2^{+/-}$neurons with anti-GluR1 (GluA1) and vGlut 1 antibodies to confirm that postsynaptic receptors are localized next to presynaptic puncta. Indeed, vGlut1 clusters were co-localized with GluA1 puncta on the dendritic shaft of $\mathrm{Tsc2}^{+/-}$neurons (Supplementary Fig. $4 \mathrm{~h}$ ), consistent with the dendritic shaft synapses in $T s c 2^{+}-$neurons being functional.

Furthermore, we noticed that the number of synaptic vesicles in the readily releasable pool (RRP) was significantly reduced by ephrinB3 siRNA in $T s c 2^{+1-}$ neurons but not in WT neurons (Fig. 6g). In this experiment, we estimated the number of synaptic vesicles in the RRP by simply dividing the hypertonic sucroseevoked RRP charge by an individual average mEPSC charge; thereby, the RRP charge should involve all synaptic releases from presynaptic terminals projecting to both spine and shaft synapses. Thus, the finding that number of releasable vesicles was reduced by ephrinB3 siRNA in $T s c 2^{+1-}$ neurons also implies that dendritic shaft synapses are functional. Because vesicular release probability was unchanged in ephrinB3-knockdown neurons, we believe that presynaptic effects can be excluded. However, because presynaptic development has been shown to be regulated by syntenin ${ }^{34}$, exploring whether presynaptic function is changed in TSC neurons will be the focus of our next study.

Syntenin knockout restores spine synapses in $T s c 2^{+/-}$neurons. To confirm the in vivo function of syntenin using other genetically defined animals, syntenin-floxed mice were generated (Fig. 7a). These mice were crossed with $\alpha C a M K I I$ Cre mice ${ }^{35}$ to obtain CaMKII ${ }^{\text {Cre }}$;syntenin flox/ $+{ }_{\left(\text {syntenin }^{+/-}\right)}$mice. Syntenin $^{+/-}$mice were born in a Mendelian ratio and could not be distinguished from their littermate controls at birth. We further crossed these mice with $T s c 2^{+/-}$mice $^{36}$ and prepared control (WT, CaMKII Cre or syntenin flox/+), syntenin ${ }^{+/-}$, $\mathrm{Tsc2}^{+/-}$and $\mathrm{Tsc2}^{+/-}$;syntenin ${ }^{+/-}$mice. We then compared ex vivo dendritic spine morphology of the hippocampal CA1 neurons in each brain slices using DiOlistic labelling. Similar to $T s c 2^{+/-}$rat neurons ${ }^{14}$, mouse $T s c 2^{+/-}$neurons showed dendritic protrusions that were longer and thinner than those of control neurons (Fig. 7b,c). By contrast, genetic heteroinsufficiency of syntenin in $T s c 2^{+/-}$neurons increased the width and reduced the length of dendritic protrusions (Fig. 7b,c). The protrusion density was also increased in $\mathrm{Tsc}^{+/-}$neurons compared with that in control or $T s c 2^{+/-} ;$syntenin ${ }^{+/-}$neurons (Fig. 7d). Syntenin ${ }^{+/-}$neurons showed narrow and thin dendritic protrusions ex vivo (Fig. $7 \mathrm{~b}, \mathrm{c}$ ).

Next, we cultured hippocampal neurons from each mouse line and compared the excitatory spine and shaft synapse densities among control, syntenin ${ }^{+/}, T s c 2^{+/-}$and $T s c 2^{+/-}$; syntenin ${ }^{+1-}$ neurons. Mouse Tsc2 ${ }^{+/-}$neurons showed a reduced spine synapse density and an increased shaft synapse density, whereas the heterozygous knockout of syntenin reversed these abnormalities in Tsc2 ${ }^{+/-}$neurons (Fig. 7e,f). Mouse syntenin $^{+/-}$neurons again showed a decrease in spine synapse density (Fig. 7e,f), as observed in syntenin-knockdown rat WT neurons (Fig. $2 \mathrm{~h}, \mathrm{i})$. To confirm these results, we electrophysiologically analysed synaptic properties of mouse control, syntenin ${ }^{+/}, T_{s c 2} 2^{+}-$and $T s c 2^{+/-}$;syntenin ${ }^{+/-}$neurons (Supplementary Fig. 5). Similar to rat $T s c 2^{+/-}$neurons, mouse $T s c 2^{+/-}$neurons showed no significant changes compared with control neurons in evoked EPSC amplitude, mEPSC amplitude, mEPSC frequency, vesicular release probability and number of releasable vesicles. Tsc2 ${ }^{+/-}$; syntenin ${ }^{+/-}$neurons also showed electrophysiological properties similar to those of control, suggesting that syntenin reduction may change the spine/shaft synapse ratio in $T s c 2^{+/-}$neurons without affecting overall excitatory synaptic transmission. However, we found that the mEPSC frequency was significantly reduced in syntenin $+/-$ neurons compared with that in $T s c 2^{+/-}$;syntenin ${ }^{+/-}$neurons. Notwithstanding, evoked EPSC amplitude, mEPSC amplitude and other presynaptic functions were unchanged between syntenin $^{+/-}$and $\mathrm{Tsc2}^{+/-} ;$syntenin ${ }^{+/-}$neurons. The reason for this decrease in syntenin $+1-$ neurons is unclear, but it may reflect the reduction in spine synapse density without increases in shaft synapse density in syntenin ${ }^{+/-}$neurons (Fig. 7e,f). Thus, genetic knockout of syntenin can restore both dendritic spine and shaft synapse densities in $T s c 2^{+/-}$mice.

\section{Discussion}

The present study suggests a novel mechanism for the regulation of excitatory spine and shaft synapse morphogenesis via Rhebsyntenin signalling. We also show that this mechanism is dysregulated in TSC neurons. Figure 8 summarizes our working

Figure 3 | Proteasomal degradation of the Rheb-syntenin complex is required for spine formation. (a) Treatment with lactacystin (Lact; $0.5 \mu \mathrm{M}$ ) for

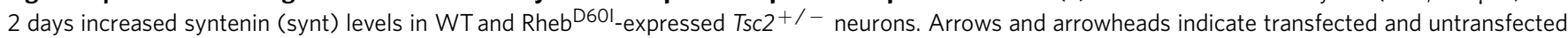
neurons, respectively. Scale bar, $10 \mu \mathrm{m}$. (b) Quantification of the syntenin levels as shown in $\mathbf{a}\left(n=10 / 3\right.$ neurons/experiments per condition, ${ }^{\star} P<0.05$, ${ }^{\star \star} P<0.01$, unpaired, two-tailed $t$-test). (c) Dendrites of untransfected, Rheb ${ }^{D 60 l}$-transfected or Rheb ${ }^{D 60 l}$ plus scrambled or syntenin siRNA-transfected WT and $T s c 2^{+/-}$neurons treated or untreated with lactacystin. Scale bar, $10 \mu \mathrm{m}$. (d) Quantification of the spine synapse density as shown in c $(n=500 / 5 / 3$ (WT, untreated), 500/5/3 (WT, treated with $0.5 \mu \mathrm{M}$ lactacystin), 500/5/3 (WT, Rheb ${ }^{\mathrm{D} 60 \mathrm{l}}$ ), 500/5/3 (WT, Rheb ${ }^{\text {D60l }}$ treated with $0.5 \mu \mathrm{M}$ lactacystin), $500 / 5 / 3$ (WT, Rheb ${ }^{\mathrm{D} 601}$ treated with $0.5 \mu \mathrm{M}$ lactacystin + syntenin siRNA), 500/5/3 (WT, Rheb ${ }^{\mathrm{D} 60 \mathrm{I}}$ treated with $0.5 \mu \mathrm{M}$ lactacystin + scrambled siRNA), 500/5/3 (Tsc2 $2^{+/-}$, untreated), 500/5/3 (Tsc2 ${ }^{+/-}$, treated with $0.5 \mu \mathrm{M}$ lactacystin), 1,029/10/5 (Tsc2 ${ }^{+/-}$, Rheb $\left.^{\mathrm{D} 60 l}\right), 1,017 / 10 / 5$ (Tsc2 ${ }^{+/-}$, Rheb ${ }^{\mathrm{D} 60 \mathrm{I}}$ treated with $0.5 \mu \mathrm{M}$ lactacystin), 500/5/3 (Tsc2 ${ }^{+/-}$, Rheb ${ }^{\mathrm{D} 60 \mathrm{I}}$ treated with $0.5 \mu \mathrm{M}$ lactacystin + syntenin $\left.s i R N A\right), 500 / 5 / 3(T s c 2+/-$ $\mathrm{Rheb}^{\mathrm{D} 60 \mathrm{I}}$ treated with $0.5 \mu \mathrm{M}$ lactacystin + scrambled siRNA) and protrusions/neurons/experiments, ${ }^{\star \star \star} P<0.001$, three-way ANOVA, post hoc Tukey test). (e) Left, western blots of syntenin (lanes 1 and 2) and Rheb (lanes 3 and 4) from untreated (top) and MG132-treated (bottom) COS-7 cells coexpressing EGFP-syntenin and EGFP-Rheb ${ }^{\mathrm{D} 60 \mathrm{I}}$ or Rheb ${ }^{\mathrm{Q} 64 \mathrm{~V}}$. (f,g) Quantification of the relative syntenin (f) and Rheb (g) levels in the absence of MG132 compared with those in the presence of MG132 shown in $\mathbf{e}\left(n=8\right.$ independent experiments, ${ }^{\star \star} P<0.01$, unpaired, two-tailed $t$-test). (h) Immunoblots (IB) of EGFP-Rheb from untreated (top) and MG132-treated (bottom) COS-7 cells expressing EGFP-Rheb ${ }^{\text {D6OI }}$ plus EGFP-syntenin (lane 1), syntenin ${ }^{\Delta C}$ (289-294) (lane 2) or syntenin ${ }^{\Delta C(295-300)}$ (lane 3). (i) Quantification of the relative Rheb levels with each syntenin mutant in the absence of MG132 compared with those in the presence of MG132 as shown in $\mathbf{h}\left(n=8\right.$ independent experiments, ${ }^{\star \star \star} p<0.001$, two-tailed $t$-test). Blots in e,h are cropped, and full-length blots are presented in Supplementary Fig. 6 h,i. All data are presented as mean \pm s.e.m. 
model in which Rheb and syntenin are essential regulators of the formation of spine and shaft synapses. During normal development, the hamartin/tuberin complex inactivates RhebGTPase, which associates with syntenin and triggers the subsequent degradation of the protein complex by the proteasome. Upon Rheb activation through Tsc mutation(s), Rheb dissociates from syntenin and binds to FKBP38 (ref. 37) and/or PLD1 (ref. 38), resulting in the activation of mTORC1.
Accumulated syntenin binds to syndecan-2 in dendritic protrusions, thereby competitively excluding CASK. Because CASK links syndecan-2 with the actin cytoskeleton via protein 4.1 (ref. 16), the elimination of CASK may destabilize the spine structure. In addition, syntenin binds to ephrinB3 in dendritic shafts and enhances excitatory shaft synapse formation. Our results indicate that the association between syndecan-2 and CASK is crucial for normal spine synapse development, and a

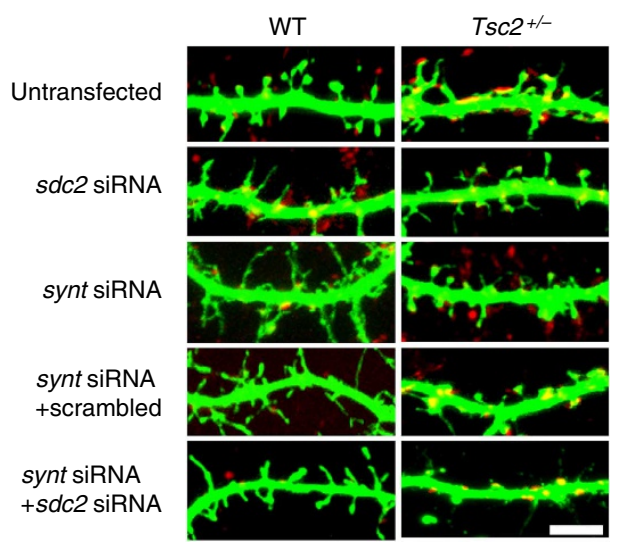

C

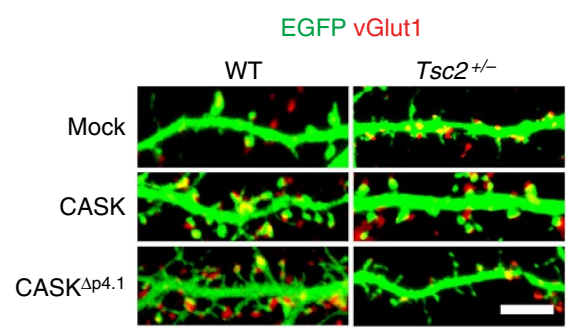

b
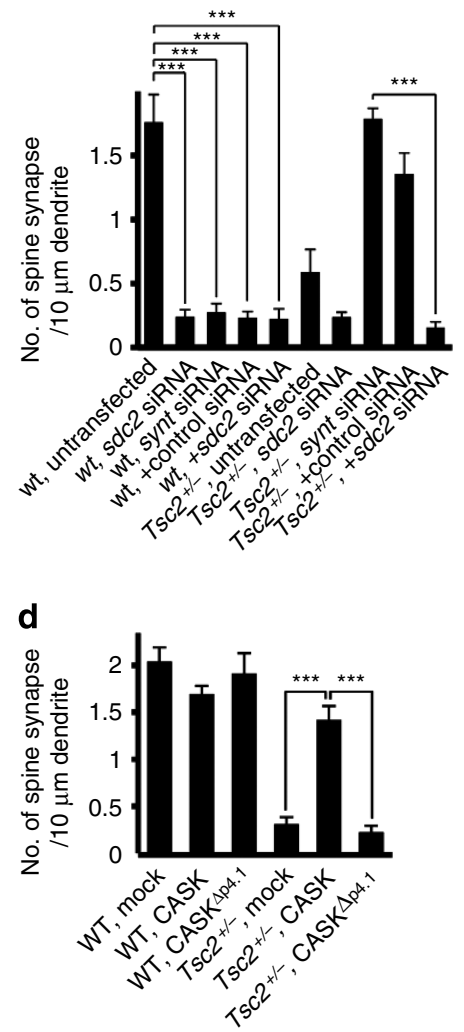

e

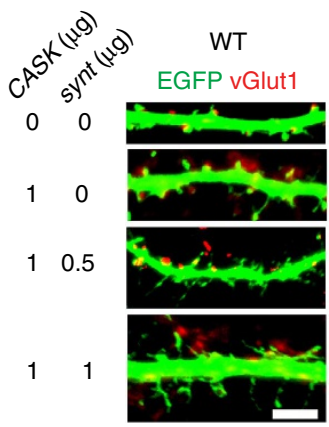

g

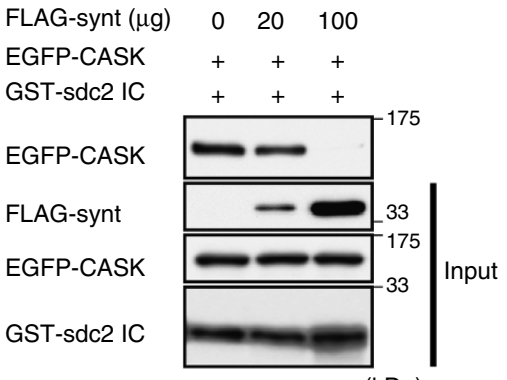

f

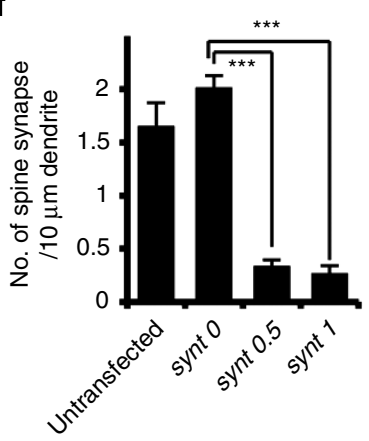

h

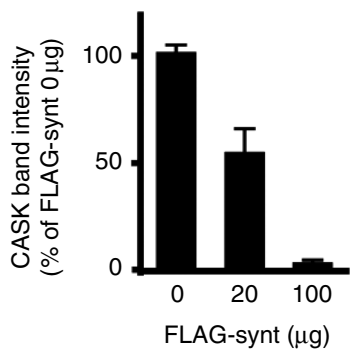


a

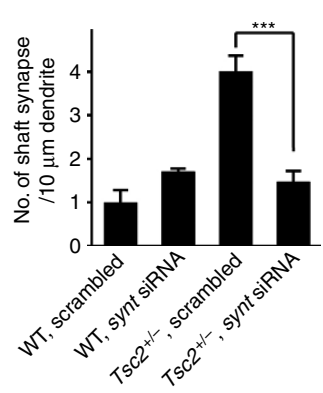

b

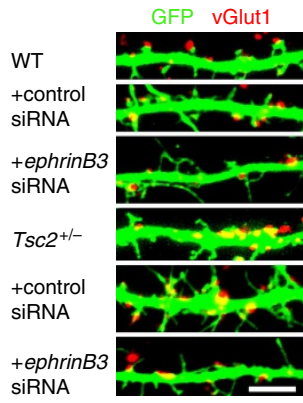

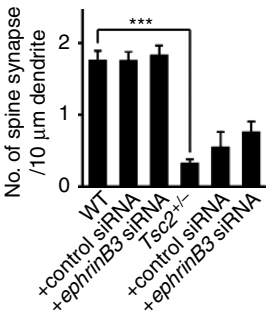

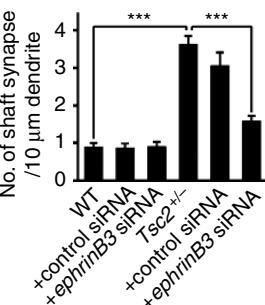

C

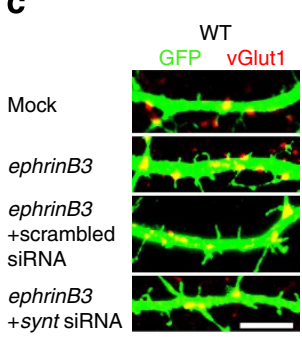

d

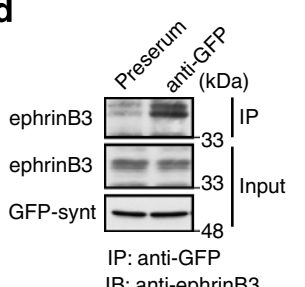

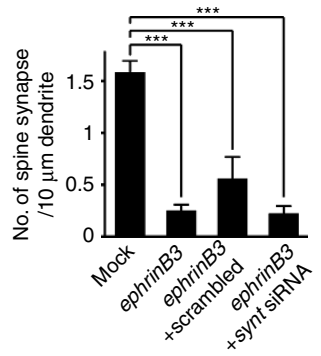

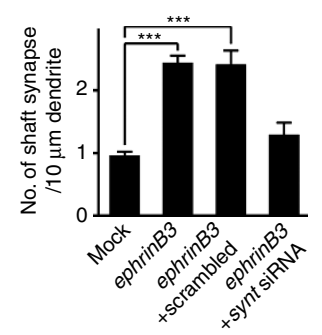

e

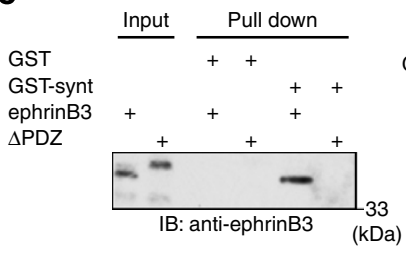

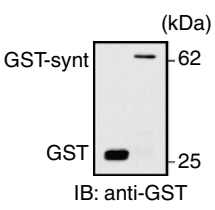

Figure 5 | Syntenin enhances excitatory shaft synapse density in the downstream of ephrinB3. (a) Quantification of vGlut1 puncta density on dendritic shafts of WT and Tsc2 ${ }^{+/}$neurons transfected with scrambled or syntenin siRNA ( $n=500 / 5 / 3$ (WT, scrambled), 500/5/3 (WT, syntenin siRNA), 1,146/10/5 ( $T s c 2^{+/-}$, scrambled) and 1,034/10/5 (Tsc2 ${ }^{+/-}$, syntenin siRNA) synapses/neurons/experiments, ${ }^{\star \star \star} P<0.001$, two-way ANOVA, post hoc Tukey test). (b) EphrinB3 siRNA decreased shaft synapse formation. (Left) EGFP plasmid and either ephrinB3 siRNA or control siRNA was expressed in WT and $T s c 2^{+/-}$neurons, and the spine and shaft synapse densities were examined. Scale bar, $10 \mu \mathrm{m}$. (Right) Quantitative analysis of the data shown in the left panel. EphrinB3 siRNA significantly reduced the shaft synapse density in Tsc2 ${ }^{+/-}$neurons $(n=500 / 5 / 3$ synapses/neurons/experiments/each condition, ${ }^{\star \star \star} P<0.001$, two-way ANOVA, post hoc Tukey test). (c) EphrinB3 increased shaft synapse density via syntenin. (Left) EphrinB3 was co-expressed in WT neurons with syntenin siRNA or scrambled siRNA, and the spine and shaft synapse densities were counted. Scale bar, $10 \mu \mathrm{m}$. (Right) Quantitative analysis of the data shown in the left panel. EphrinB3-induced shaft synapse increase was inhibited by syntenin siRNA but not by scrambled siRNA ( $n=500 / 5 / 3$ synapses/neurons/experiments/each condition, ${ }^{\star \star \star} P<0.001$, two-way ANOVA, post hoc Tukey test). (d) Co-immunoprecipitation of ephrinB3 with EGFP-syntenin from HEK293T cells expressing both proteins using anti-GFP antibody. (e) PDZ-dependent binding of syntenin to ephrinB3. EphrinB3- or ephrinB3 ${ }^{\triangle P D Z}$-expressing cell lysates were pulled down with GST-syntenin. The precipitates were immunoblotted (IB) with anti-ephrinB3 antibody. Blots in $\mathbf{d}, \mathbf{e}$ are cropped and full-length blots are presented in Supplementary Fig. 6k,l. All data are presented as mean \pm s.e.m.

Figure 4 | Syntenin prevents spine formation by competing with CASK for binding to syndecan-2. (a) Dendrites of WT and Tsc2 $+/-$ neurons either untransfected or transfected with syndecan-2 (sdc2) siRNA, syntenin (synt) siRNA, syntenin siRNA plus scrambled siRNA or syntenin siRNA plus syndecan-2. Scale bar, $10 \mu \mathrm{m}$. (b) Quantification of spine synapse density as shown in a ( $n=500 / 5 / 3$ (WT, untransfected), 500/5/3 (WT, syndecan-2 siRNA), 500/5/3 (WT, syntenin siRNA), 500/5/3 (WT, syntenin siRNA + scrambled siRNA) and 500/5/3 (WT, syntenin siRNA + syndecan-2 siRNA) protrusions/ neurons/experiments, 500/5/3 (Tsc2 ${ }^{+/}$, untransfected), 500/5/3 (Tsc2 ${ }^{+/-}$, syndecan-2 siRNA), 500/5/3 (Tsc2 ${ }^{+/-}$, syntenin siRNA), 893/10/5 $\left(T_{s c 2}{ }^{+/-}\right.$, syntenin siRNA + scrambled siRNA) and 1,196/10/5 (Tsc2 ${ }^{+/-}$, syntenin siRNA + syndecan-2 siRNA) protrusions/neurons/experiments, ${ }^{\star \star \star} P<0.001$, two-way ANOVA, post hoc Tukey test). (c) Dendrites of mock-, CASK- or CASK ${ }^{\mathrm{p} 4.1}$-transfected WT and Tsc2 ${ }^{+/}-$neurons. Scale bar, $10 \mu \mathrm{m}$. (d) Quantification of spine synapse density shown in c $\left(n=500 / 5 / 3\right.$ (WT, mock), 500/5/3 (WT, CASK), 500/5/3 (WT, CASK $\left.{ }^{\Delta p 4.1}\right), 1,023 / 10 / 5$ (Tsc2 ${ }^{+/-}$, mock), 1,070/10/5 (Tsc2 ${ }^{+/-}$, CASK) 1,040/10/5 (Tsc2 $2^{+}$- , CASK ${ }^{\Delta \mathrm{p} 4.1}$ ) protrusions/neurons/experiments, ${ }^{\star \star \star} P<0.001$, unpaired, two-way ANOVA, post hoc Tukey test). (e) Dendrites of WT hippocampal neurons transfected with EGFP or EGFP-CASK plus $0,0.5$ or $1 \mu \mathrm{g}$ of ECFP-syntenin were immunolabelled with anti-GFP and anti-vGlut1 antibodies. Scale bar, $10 \mu \mathrm{m}$. (f) Quantification of spine synapse densities as shown in e $(n=500 / 5 / 3$ (CASK 0, synt 0), 1,014/10/5 (CASK 1, synt 0), 953/10/5 (CASK 1, synt 0.5) and 1,152/10/5 (CASK 1, synt 1) protrusions/neurons/experiments, ${ }^{\star \star \star} \mathrm{P}<0.001$, two-tailed $t$-test). (g) Pull-down assays showing that FLAG-syntenin inhibits the binding of EGFP-CASK to the IC of syndecan-2 (GST-sdc2 IC) in a dose-dependent manner. These blots are cropped and full-length blots are presented in Supplementary Fig. 6j. (h) Quantification of EGFP-CASK pulled down with GST-sdc2 IC in the presence of increasing amount of FLAG-syntenin ( $n=7$ independent experiments). All data are presented as mean \pm s.e.m. 
a

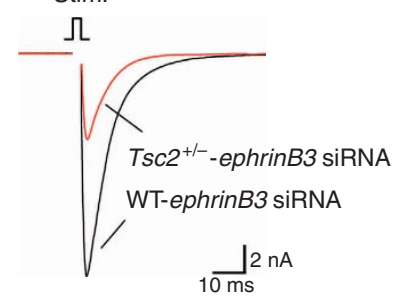

b

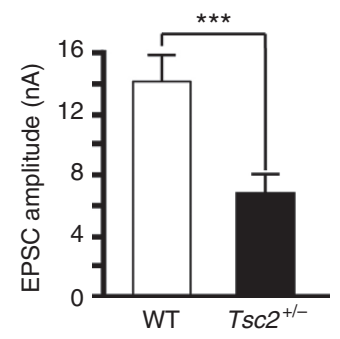

f

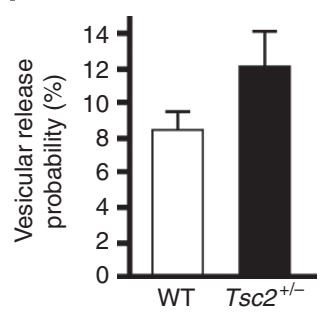

c

WT-ephrinB3 siRNA
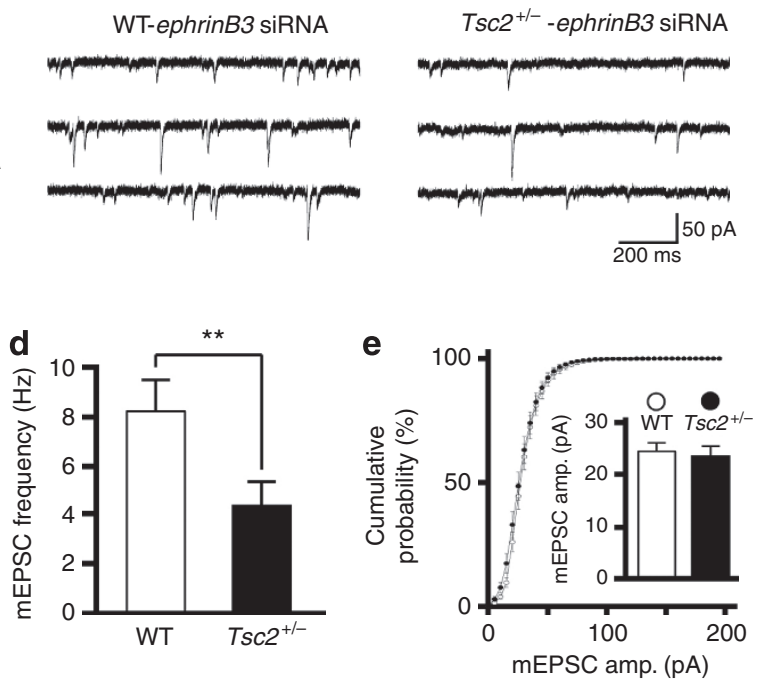

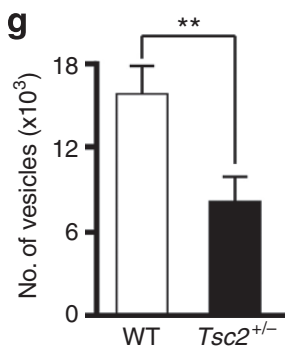

Figure 6 | Synaptic transmission in Tsc2 ${ }^{+/-}$neurons is functionally compensated for by ephrinB3-mediated shaft synapses. (a) Representative

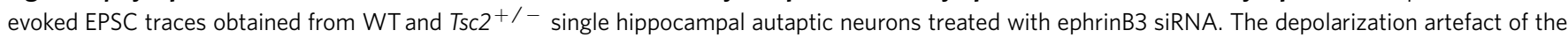
action potential evoked by electrical stimulation (Stim.) was blanked. (b) Mean EPSC amplitude in WT ( $n=36)$ and Tsc2 $+/-(n=34)$ autaptic neurons treated with ephrinB3 siRNA ( ${ }^{\star \star} P<0.001$ ). (c) Representative mEPSC traces in WT and Tsc2 ${ }^{+} /-$autaptic neurons treated with ephrinB3 siRNA. (d) Mean mEPSC frequency in WT $(n=34)$ and Tsc2 $+/-(n=35)$ autaptic neurons treated with ephrinB3 siRNA ( $\left.{ }^{\star \star} P<0.01\right)$. (e) Cumulative probability plots of the mEPSC amplitude (amp.) in WT $(n=34)$ and Tsc2 ${ }^{+/-}(n=35)$ autaptic neurons treated with ephrinB3 siRNA. The inset shows the mean mEPSC

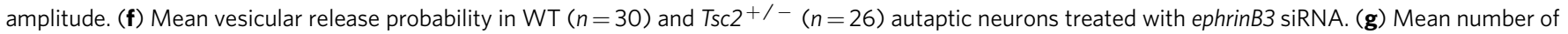

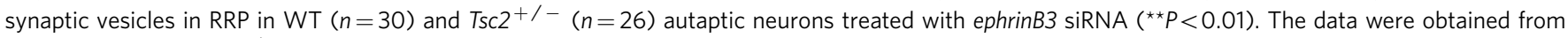
21 (WT) and 14 (Tsc2 ${ }^{+/-}$) experiments, respectively. All data are presented as mean \pm s.e.m. $\left({ }^{\star \star} P<0.01\right.$, ${ }^{\star \star \star} P<0.001$, Student's $t$-test).

the disruption of this interaction by syntenin may cause abnormalities in dendritic spine and shaft synapses.

Consistent with a human Golgi study ${ }^{12}$, mouse models of TSC have demonstrated dendritic spine abnormalities ${ }^{13,39}$. In particular, Tavazoie et al. ${ }^{13}$ showed that Tscl deficiency in cultured hippocampal slices led to decreased spine density, increased spine length and head width. These findings are somewhat different from our observations (decreased spine synapse density, increased protrusion length and decreased protrusion width in dissociated $T s c 2^{+/-}$neurons). This discrepancy may reflect differences in culture conditions (slice culture versus dissociated culture) and/or neuronal maturation. However, the common finding is that these spine abnormalities were not restored by inhibition of mTORC1. We previously found that suppression of Rheb-GTPase activity with farnesyl transferase inhibitors almost completely rescued spine synapse formation ${ }^{14}$. Thus, a non-canonical pathway such as this Rheb-syntenin signalling may participate in some of the symptomatic manifestations in TSC $^{40-42}$. Indeed, knockdown and knockout of brain syntenin restored contextual fear discrimination deficits in $T s c 2^{+/-}$rats and mice, respectively (unpublished data). In addition, to confirm our findings using human neurons, we will generate mutations in Tsc gene in human-induced pluripotent stem cells for differentiation into excitatory neurons. These future results may provide new mechanistic insights into the pathogenesis of neurological symptomatic manifestations in TSC patients. Furthermore, development of a method to control Rheb activity in living neurons may strengthen a direct link between the Rheb-syntenin signalling and spine formation.

We show that increased syntenin diminished spine synapse density in TSC neurons, whereas a previous study demonstrated that syntenin overexpression in WT neurons did not reduce spine synapse density ${ }^{30}$. This discrepancy may be caused by the use of different antibodies to detect presynaptic terminals; we immunolabelled only excitatory synapses with anti-vGlut1, whereas $\mathrm{Xu}$ et al. ${ }^{30}$ used an anti-synapsin antibody that reacts with both excitatory and inhibitory terminals. Because inhibitory synapses were also increased in TSC neurons (unpublished data), the use of an anti-synapsin antibody might produce different results from this study. The other difference between this study and that by $\mathrm{Xu}$ et $\mathrm{al}^{30}$ is in the mEPSC frequency in ephrinB3-reduced neurons. We found no significant change in the mEPSC frequency in ephrinB3-knockdown WT neurons (Fig. 6d and Supplementary Fig. 4d), whereas Xu et al. ${ }^{30}$ showed a clear decrease in mEPSC frequency in ephrinB3-deficient neurons ${ }^{30}$. This discrepancy may be because of the differences in neuronal maturation. We used cultured hippocampal neurons at DIV 14-23, whereas Xu et al. ${ }^{30}$ euthanized P12 mice for electrophysiology. Excitatory synapses translocate from dendritic 
a
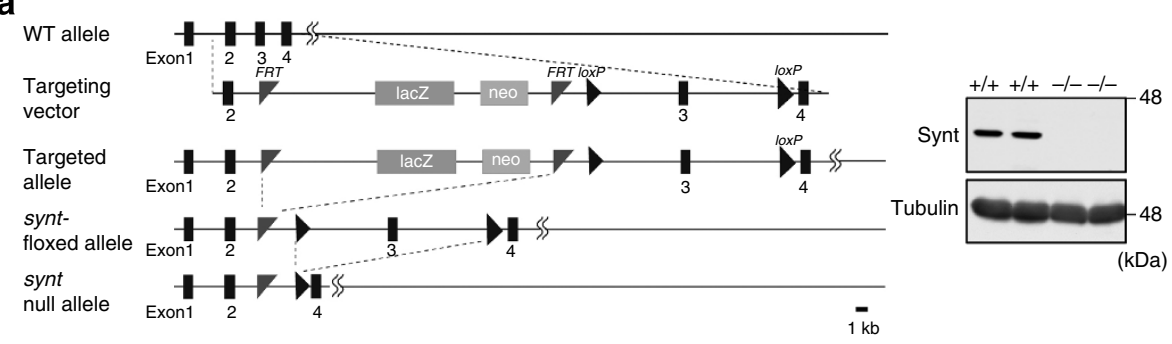

b

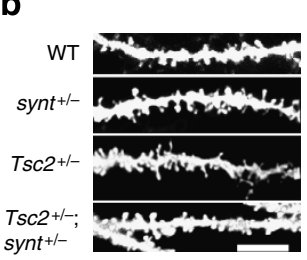

C

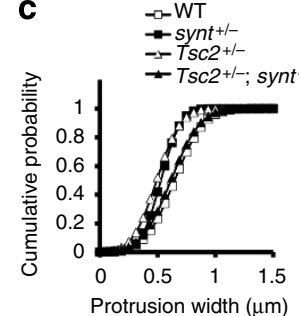

$\overline{1 \mathrm{~kb}}$

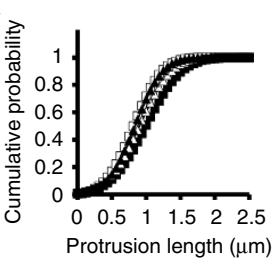

d

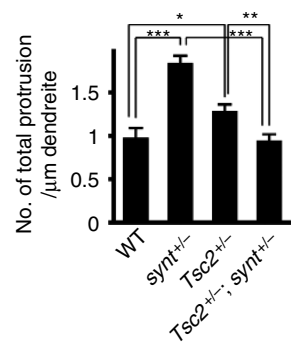

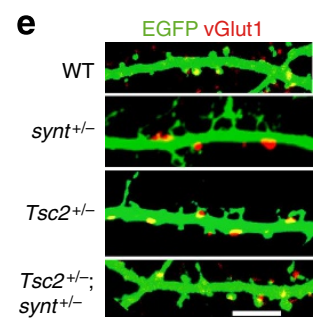

f
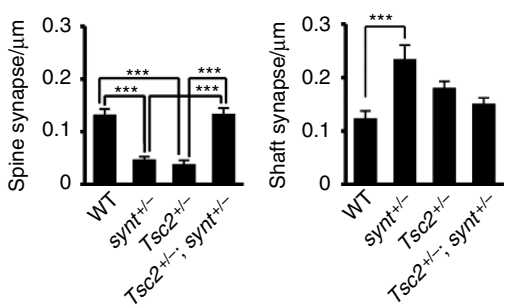

Figure 7 | Heterozygous knockout of syntenin rescued spine dysmorphogenesis in Tsc2 ${ }^{+} /{ }^{-}$mice. (a) Targeting construct for the generation of synteninfloxed mice. In the targeting vector, exon 3 of the syntenin gene was flanked by LoxP sites, indicated with black triangles. The neomycin resistance cassette was surrounded by flippase recognition target sequences, shown as black rectangles (left). Immunoblotting of WT and syntenin knockout brain lysates with anti-syntenin antibody (right). These blots are cropped and full-length blots are presented in Supplementary Fig. 6m. (b) Representative Dil-labelled dendrites of hippocampal CA1 neurons from control, syntenin ${ }^{+/-}, T s c 2^{+/-}$and $T s c 2^{+/-}$;syntenin ${ }^{+/-}$mice. Scale bar, $10 \mu \mathrm{m}$. (c) Cumulative probability plots of the protrusion widths (left) and lengths (right) as shown in $\mathbf{b}$. $T s c 2^{+/-}$and syntenin ${ }^{+/-}$neurons showed decreased protrusion width $\left(D=0.2706, P<0.001\left(T s c 2^{+/-} ;\right.\right.$;syntenin ${ }^{+/-}$and $\left.T s c 2^{+/}{ }^{-}\right), D=0.2417, P<0.001\left(T_{s c 2}{ }^{+/-}\right.$; syntenin ${ }^{+/}$and syntenin $\left.{ }^{+/-}\right), D=0.4069$, $P<0.001$ (control and $T s c 2^{+/-}$) $, D=0.3042, P<0.001$ (control and syntenin ${ }^{+} /-$)) and an increased protrusion length $(D=0.0885, P<0.05$

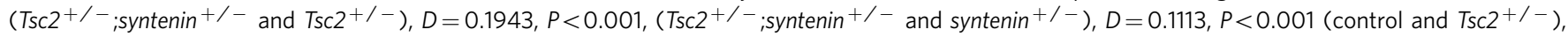
$D=0.2117, P<0.001$ (control and syntenin ${ }^{+/-}$)) compared with $T_{s c 2^{+/}}{ }^{-} ;$syntenin $^{+/-}$and control neurons, respectively. ssc2 $^{+/-} ;$syntenin ${ }^{+/-}$ neurons exhibit dendritic protrusions almost identical to those of control neurons. Heterozygous knockout of syntenin rescued the aberrant protrusion shape in $T s c 2^{+/-}$mice $\left(n=994 / 10 / 5\right.$ (control), 652/6/4 (syntenin $\left.{ }^{+/-}\right), 1,073 / 10 / 5$ (Tsc2 $2^{+/-}$) and 1,043/10/5 (Tsc2 $2^{+/-}$;syntenin ${ }^{+/-}$) protrusions/ neurons/experiments, Kolmogorov-Smirnov test). (d) Quantification of the protrusion density in $\mathbf{b}$. Tsc2 ${ }^{+/-}$and syntenin $+/-$neurons exhibited higher protrusion densities than $T s c 2^{+/-}$; syntenin ${ }^{+/-}$and control neurons, respectively $\left({ }^{\star} P<0.05,{ }^{\star \star} P<0.01,{ }^{\star \star \star} P<0.001\right.$, two-way ANOVA, post hoc Tukey test). (e) $T s c 2^{+/-}$mouse neurons showed a decrease in excitatory (vGlut1-positive) spine synapse density and an increased in shaft synapse density, and this phenotype was rescued by heterozygous knockout of syntenin. Syntenin ${ }^{+/-}$neurons also showed decreased spine synapse density and increased shaft synapse density. Scale bar, $10 \mu \mathrm{m}$. (f) Data quantification in e. All data are presented as mean \pm s.e.m. ( $n=500 / 5 / 3$ synapses $/$ neurons/experiments/each condition, ${ }^{\star \star \star} P<0.001$, two-way ANOVA, post hoc Tukey test).

shafts (shaft synapses) to spines (spine synapses) during neuronal development ${ }^{43}$. We used mature neurons with fewer shaft synapses, which are the main targets of regulation by ephrinB3 (ref. 32). This may explain why ephrinB3 knockdown failed to reduce synaptic transmission in this study.

Reducing the syntenin levels in WT neurons also decreased the spine synapse density (Fig. 2h,i). Thus, the deviation of syntenin levels from a narrow physiological range may disrupt spine synapse formation. In contrast to $T s c 2^{+\%}-$ neurons, however, syntenin $^{+/-}$neurons did not show increased shaft synapse density (Fig. 7f), suggesting that syntenin reduction may affect only spine synapses. How the reduction in syntenin levels decreases spine synapse density remains unclear; however, it is noteworthy that syntenin associates with multiple proteins, such as adhesion molecules $\left(\right.$ SynCAM $^{44}$, neurexin and neurofascin ${ }^{45}$ ) or cytoplasmic proteins (PICK1; ref. 46). The absence of these associations may contribute to impaired spine formation. It is possible that such distinct syntenin function regulates dendritic spinogenesis in different ways. Clarification of this mechanism will be the next step in understanding the multiple functions of syntenin.

CASK is also a scaffold protein that interacts with $>20$ of binding partners through its PDZ domain. In postsynaptic neurons, both syntenin and CASK can bind to syndecan-2, which regulates dendritic spine formation ${ }^{26}$. We show that the increase in syntenin levels may impair spine synapse morphogenesis in $T s c 2^{+/-}$neurons. Such synaptic abnormalities in TSC neurons may be a cause of mental retardation and/or refractory epilepsy in early childhood, called West syndrome ${ }^{5}$. In addition, CASK gene mutations cause intractable epilepsy and intellectual disability in infancy ${ }^{47}$, called Ohtahara syndrome ${ }^{48}$. Ohtahara syndrome often evolves into West syndrome, therefore these two syndromes are 


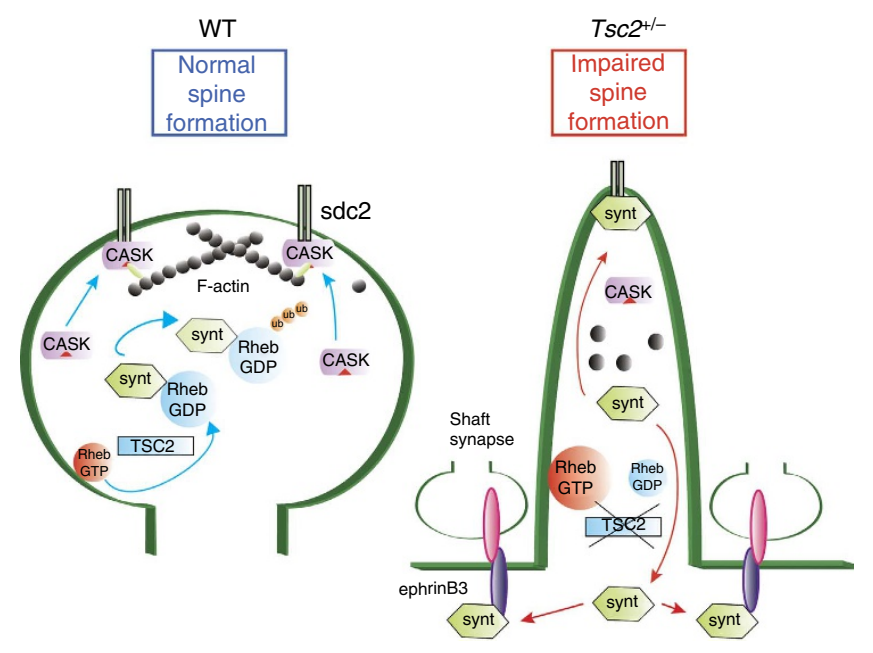

Figure 8 | Proposed model for the role of Rheb and syntenin in spine and shaft synapse formation. During normal development, Tsc2 (tuberin) converts Rheb-GTP into inactive Rheb-GDP. Rheb-GDP binds to syntenin, thereby promoting syntenin degradation. This decrease in syntenin levels may facilitate the association of CASK with syndecan-2 (sdc2) and the formation of spinal F-actin, leading to normal spine development (left, WT). However, Tsc2 disruption in tuberous sclerosis increases Rheb-GTP, which rescues syntenin from proteasomal destruction. Accumulated syntenin may inhibit the binding of CASK to sdc2 and promote its binding to ephrinB3, resulting in impaired spine morphogenesis and enhanced shaft synapse formation (right, $\mathrm{Tsc}^{+/-}$).

both classified as 'epileptic encephalopathy'. Thus, the increases in syntenin levels and the CASK mutations may cause related neurodevelopmental disorders, suggesting that the two proteins functionally compete against each other.

Here, we focused on the contributions of postsynaptic syntenin and CASK to the control of spine formation; however, both proteins are also localized to presynaptic terminals and bind to $\beta$-neurexin ${ }^{49,50}$. To examine the presynaptic effects of Tsc2 mutations on excitatory synapse formation, we compared the size of vGlut1 puncta in WT-WT synapses and $T s c 2^{+/-}-\mathrm{WT}$ synapses; however, we found no significant differences $\left(0.11 \pm 0.013 \mathrm{~mm}^{2}\right.$ for WT and $0.10 \pm 0.011$ for Tsc2 $2^{+/-}$ boutons). Because syntenin knockdown in postsynaptic TSC neurons results in spine synapse formation with presynaptic TSC neurons, spine formation may be regulated by the postsynaptic balance between syntenin and CASK rather than by presynaptic effects. Future studies are needed to characterize the effects of presynaptic syntenin and CASK on spine and shaft synapse formation.

In this study, we found that Rheb interacted with syntenin and formed a novel part of the TSC-Rheb pathway, which may help us to better understand the pathophysiology of neurological symptoms in TSC. Several preclinical studies and early human case reports have demonstrated that MTORC1 inhibitors such as sirolimus or everolimus are useful for treating seizures $39,51-54$, whereas more recent prospective trials have shown that benefits could not be demonstrated when seizure frequency was analysed as a secondary outcome measure ${ }^{55,56}$. Moreover, $10-20 \%$ of TSC patients showed exacerbation of seizures after everolimus treatment, even in the clinical trial that demonstrated the efficacy of everolimus ${ }^{57}$. Thus, in addition to mTORC1 inhibitors, new approaches for the treatment of neurological symptoms in TSC patients are needed to improve not only seizure control but also cognitive function and patient quality of life. Because Rheb expression is regulated by neuronal activity ${ }^{58}$,
Rheb-syntenin signalling may be involved in activity-dependent learning and memory processes. Appropriate modulation of this signalling pathway can be required for normal cognitive function. Thus, the Rheb-syntenin pathway may also be a therapeutic target for relieving TSC and other cognitive disorders involving abnormalities of spine and shaft synapses.

\section{Methods}

Antibodies and reagents. Antibodies used for western blotting are summarized in Supplementary Table 1. Rapamycin, MG132 and lactacystin were purchased from LKT Laboratories, Millipore and the Peptide Institute, respectively. The siRNAs used in this study are summarized in Supplementary Table 2. Oligonucleotides were synthesized and duplexed by Invitrogen (Stealth, syntenin and syndecan-2) and Dharmacon (Accell, ephrinB3). Their suppressive activities were confirmed in rat neurons and HEK293T cells using immunostaining and western blotting, respectively.

Animals. The Eker $\left(T s c 2^{+/-}\right)$and WT genotypes were determined using $\mathrm{PCR}^{59}$ Syntenin-floxed mice were obtained from the Knock-Out Mouse Project (www.komp.org; project no. CSD26463). CaMKII ${ }^{\text {Cre }}$ mice $^{35}$ were obtained from European Mouse Mutant Archive. Tsc2 ${ }^{+/-}$mice were generated previously ${ }^{36}$ Mice were maintained on a C57BL/6 background. All of the animal experiments were approved by the Animal Care and Use Committees of the Tokyo Metropolitan Institute of Medical Science and Fukuoka University.

Molecular cloning and plasmid construction. The full-length of rat Rheb was subcloned into a yeast expression vector, pPC97 (ref. 60), in a fusion complex with the GAL4 DNA-binding domain. This construct was transformed in yeast strain PCY2 (ref. 60). Transformants were selected on leucine-deficient ( Leu $^{-}$) medium and subsequently transformed with an oligo(dT)-primed rat hippocampal cDNA library subcloned into pPC86 (ref. 61). Positive colonies were selected on tripledeficient plates $\left(\mathrm{Leu}^{-}, \mathrm{Trp}^{-}\right.$and adenine ${ }^{-}$) and were assayed for $\beta$-galactosidase activity. Their plasmids were isolated and transformed into Escherichia coli. The E. coli clones containing the pPC86 construct were rescued and their plasmids were isolated and sequenced. For expression in primary neurons, COS-7 cells and HEK293T cells, rat Rheb, syntenin and syndecan-2 cDNAs were cloned into the EGFP-C3, ECFP-C3 and pEF-FLAG vectors, respectively. Rat CASK and Rheb cDNAs were cloned into ECFP-C3 and pEF-FLAG, respectively. EphrinB3 cDNA was cloned into pCAG. The syntenin ${ }^{\Delta N(1-115)}$ mutant was generated using PCR from rat syntenin cDNA. Using the QuikChange Site-Directed Mutagenesis Kit (Stratagene), Rheb mutants (D60I, T38M/D60I, I39K/D60I and Q64V), syntenin mutants (mPDZ1, mPDZ2, $\triangle \mathrm{C}(278-300), \Delta \mathrm{C}(289-300), \Delta \mathrm{C}(289-294)$ and $\Delta \mathrm{C}(295-300))$, a $C A S K$ mutant $(\Delta 4.1)$ and an ephrinB3 mutant ( $\triangle \mathrm{PDZ})$ were generated from WT Rheb, syntenin, CASK and ephrinB3, respectively ${ }^{27,49,62}$. The siRNA-resistant forms of the syntenin mutants and ephrinB3 were generated through the introduction of silent mutations in the syntenin and ephrinB3 siRNA target sequences, respectively.

Immunoprecipitation. Rat brains, COS-7 cells and HEK293T cells were homogenized with lysis buffer (20 mM Tris- $\mathrm{HCl}$ ( $\mathrm{pH} 8.0$ ), $0.15 \mathrm{M} \mathrm{NaCl}, 1 \mathrm{mM}$ EDTA, $1 \%$ Triton X-100, protease inhibitor cocktail tablet (Roche) and $1 \mathrm{mM}$

phenylmethylsulfonyl fluoride). The homogenates were centrifuged to obtain clear protein extracts. Anti-syntenin (goat, Santa Cruz Biotechnology), anti-GFP (rabbit, Invitrogen) or anti-FLAG (mouse, Sigma-Aldrich) antibodies were added to the protein extracts on ice for $1 \mathrm{~h}$, and the mixtures were incubated overnight at $4{ }^{\circ} \mathrm{C}$ with packed protein G Sepharose (GE Healthcare). The precipitated immune complex was boiled in SDS sample buffer and analysed using western blotting with anti-Rheb (rabbit, CST), anti-FLAG (mouse, Sigma-Aldrich), anti-GFP (rabbit, Invitrogen) or anti-ephrinB3 (rabbit, Invitrogen) antibodies ${ }^{63}$. All representative western blots included in the manuscript are shown as full scans in Supplementary Fig. 6.

Recombinant protein expression and pull-down assay. For the bacterial expression of recombinant GST-tagged syntenin and the syntenin mutants, the corresponding sequences were cloned into the pGEX-4T2 vector (GE Healthcare) and expressed in BL21(DE3) cells. Each GST-fused syntenin protein was purified with glutathione beads (GE Healthcare). To examine the binding between Rheb and syntenin, the same amount of purified GST-syntenin (WT or mutant) was first attached to glutathione beads and then incubated with cell extracts expressing various versions of enhanced green fluorescent protein (EGFP)-tagged Rheb overnight at $4{ }^{\circ} \mathrm{C}$. To determine whether the nucleotide-binding state of Rheb affects the binding of syntenin to the IC of syndecan-2, the purified GST-syndecan2 IC protein was first attached to glutathione beads and then incubated with cell extracts expressing FLAG-tagged syntenin and either EGFP-Rheb ${ }^{\text {D60I }}$ or EGFPRheb ${ }^{\mathrm{Q} 64 \mathrm{~V}}$ overnight at $4^{\circ} \mathrm{C}$. To examine the binding between syntenin and ephrinB3, the purified GST-syntenin was first incubated with cell extracts expressing ephrinB3 or $\triangle \mathrm{PDZ}$ overnight at $4^{\circ} \mathrm{C}$. In all pull-down experiments, the 
beads were washed with lysis buffer and boiled in SDS sample buffer. The precipitates were analysed using western blotting with anti-GFP, anti-FLAG or antiephrinB3 antibodies. All representative western blots included in the manuscript are shown as full scans in Supplementary Fig. 6.

Cell culture and transfection. All cells were cultured at $37^{\circ} \mathrm{C}$ and $5 \% \mathrm{CO}_{2}$. Neuronal cultures were prepared from hippocampi at embryonic day 19-21 for Eker rats and at postnatal day $0-1$ for control (WT, CaMKII ${ }^{\text {Cre }}$ or syntenin flox/ + ), syntenin ${ }^{+/-}, T s c 2^{+1-}$ and $T s c 2^{+1-}$; syntenin ${ }^{+/-}$mice. Cells were plated at a density of 5,400 cells per $\mathrm{cm}^{2}$ onto polyethyleneimine-coated dishes. Primary cultures were maintained in neurobasal medium supplemented with B27, gentamicin and GlutaMAX. The neurons were transfected with expression plasmids and/or siRNAs at DIV 11-12 using Lipofectamine 2000 (Invitrogen) ${ }^{64}$. The transfection of ephrinB3 siRNA into primary neurons was performed according to the Thermo Scientific Dharmacon Accell siRNA Delivery Protocol (Thermo Scientific).

HEK293T and COS-7 cells were obtained from R. Takahashi (Riken) and T. Haga (University of Tokyo), respectively. Both cells were plated at $70-80 \%$ confluence onto polyethyleneimine-coated dishes and cultured in DMEM supplemented with $10 \%$ fetal bovine serum. Cells were transfected with plasmids using Lipofectamine 2000 (Invitrogen) ${ }^{63}$. To quantify the proteasome degradation of Rheb and syntenin proteins, Rheb- and syntenin-coexpressing COS-7 cells were untreated or treated with $20 \mu \mathrm{M}$ MG132 for $20 \mathrm{~h}$. Cultured hippocampal neurons were treated with $0.5 \mu \mathrm{M}$ lactacystin for 2 days (DIV 17-19) or $100 \mathrm{nM} \mathrm{MG132}$ for 1 day (DIV 18-19). Cell lysates were immunoblotted with anti-GFP.

Immunostaining. Rat cultured hippocampal neurons were fixed with $4 \%$ paraformaldehyde, blocked, permeabilized with BL solution (3\% normal goat serum or $3 \%$ bovine serum albumin with $0.1 \%$ Triton X-100 in PBS) and incubated overnight at $4{ }^{\circ} \mathrm{C}$ with one of the following primary antibodies: anti-syntenin (mouse, Santa Cruz Biotechnology), anti-GFP (chicken, Millipore), anti-vGlut1 (guinea pig, Millipore) or anti-ephrinB3 (rabbit, Invitrogen). The immunoreactivity was visualized using species-specific, fluorochrome-conjugated secondary antibodies.

For spine and shaft synapse density analysis, dendrites were chosen randomly and their lengths were measured in two-dimensional projection of threedimensional image stacks. Spines were defined as any protrusions from the dendritic shaft $<8 \mu \mathrm{m}$ in length ${ }^{32}$. The number of vGlutl puncta on spine and shaft synapses of the dendritic segment is determined by counting the number of vGlutl puncta that partially overlap with the GFP-positive shaft or spines. Any vGlut1 puncta that are not touching the GFP-positive dendrite are not included.

Image acquisition and quantification. Confocal images of neurons were obtained using a Zeiss $63 \times$ lens (numerical aperture 1.4) with sequential acquisition settings at the resolution of the confocal microscope $(512 \times 512$ pixels $)$. Each image was a composite constructed from a series of images captured throughout the $z$ axis of each cell. The parameters of each composite image were optimized for the particular lens and pinhole setting. Identical confocal microscope settings were maintained for all scans in each experiment. All morphometric analyses and quantifications were performed using MetaMorph image analysis software (Universal Imaging). For the spine morphology studies, individual dendritic protrusions were manually traced, and the maximum length and head width of each dendritic protrusion were measured and recorded in Microsoft Excel. To measure the average pixel fluorescence intensity of syntenin, the neurons were carefully traced and the background intensity was subtracted from the intensity in the traced regions. Neurons were selected blindly based on the GFP or MAP2 fluorescence.

Autaptic culture. Astrocytes were obtained from cerebral cortices of newborn (P0) ICR mice (Kyudo). Cerebral cortices were removed from the brains, placed in cold Hank's Balanced Saline Solution (Invitrogen) and dissociated using 0.05\% trypsinEDTA (Wako). The cells were plated in plating medium composed of Dulbecco's modified Eagle medium with GlutaMax and pyruvate (DMEM, Invitrogen) supplemented with $10 \%$ fetal bovine serum (Invitrogen) and $0.1 \%$ MITO + Serum Extender (BD Biosciences) in $75 \mathrm{~cm}^{2}$ culture flasks $(250 \mathrm{ml}$, BD Falcon). The next day, the culture flask was gently rinsed once with plating medium to remove nonadherent cells. After 2 weeks, when the glial cells had almost reached confluence, microglia and other small cells were isolated and removed by tapping the culture flask several times. The remaining astrocytes adhered to the bottom of the culture bottle were subsequently trypsinized and plated onto microdot-coated coverslips (see above) in the plating medium at a density of 6,000 cells per $\mathrm{cm}^{2}$. Once microislands were uniformly established on the coverslip, within a week, an antimitotic agent $\left(8 \mu \mathrm{M} 5\right.$-fluoro- $2^{\prime}$-deoxyuridine (Sigma-Aldrich) and $20 \mu \mathrm{M}$ uridine (Sigma-Aldrich)) was added to curtail glial proliferation and maintain the purity of the microisland cultures.

The microisland culture plates were established a few days before the neuronal cultures. The autaptic cultures of rat E18-19 and mouse P0-1 hippocampal neurons were generated ${ }^{65-67}$. Briefly, CA1-CA3 hippocampi were isolated and enzymatically dissociated in papain (2 units per ml, Worthington) in DMEM for $60 \mathrm{~min}$ at $37^{\circ} \mathrm{C}$. The dissociated hippocampal neurons were plated at a density of
1,500 cells per $\mathrm{cm}^{-2}$ onto the above astrocyte island plates. Before plating the dissociated hippocampal neurons, the astrocyte-conditioned media was replaced with Neurobasal-A medium (Invitrogen) supplemented with $2 \%$ B27 supplement (Invitrogen) and GlutaMax. The medium was not changed during the neuronal culture periods.

Electrophysiology. All synaptic recordings were undertaken on autaptic cultures after genotyping. In all cases, the synaptic responses from autaptic neurons were recorded in the whole-cell configuration under the voltage-clamp mode at a holding potential $(\mathrm{Vh})$ of $-70 \mathrm{mV}$ (MultiClamp 700B, Molecular Devices) and room temperature. The patch pipette resistance was 3-6M $\mathrm{M}$, and the series resistance was compensated by $70-90 \%$. The $\alpha$-amino-3-hydroxy-5-methyl-4-isoxazolepropionic acid (AMPA) receptor-mediated evoked glutamatergic synaptic transmission was recorded in response to an action potential elicited by a brief ( $2 \mathrm{~ms}$ ) somatic depolarization pulse (to $+0 \mathrm{mV}$ ) from the patch pipette. Spontaneous glutamatergic mEPSCs were recorded in the

presence of $0.5 \mu \mathrm{M}$ tetrodotoxin. The size of the RRP of synaptic vesicles was estimated using a synaptic transient charge elicited by a 10 -s application of $0.5 \mathrm{M}$ sucrose. The number of synaptic vesicles in the RRP was subsequently calculated by dividing the RRP charge by the individual average mEPSC charge. The vesicular release probability was calculated as the ratio of the charge of the evoked EPSC to the RRP charge successfully obtained from the same neuron. The synaptic responses were recorded at a sampling rate of $20 \mathrm{kHz}$ and filtered at $10 \mathrm{kHz}$. The data were excluded from the analysis if a leak current $>300 \mathrm{pA}$ was observed. The template for the mEPSC data analysis was set as a rise time constant of $0.5 \mathrm{~ms}$, a decay time constant of $4 \mathrm{~ms}$, a baseline of $5 \mathrm{~ms}$, and a template length of $10 \mathrm{~ms}$. The data were analysed offline using Axograph X 1.2 software (AxoGraph Scientific). Recordings of synaptic responses were performed at DIV 14-23 and DIV 13-16 from rat and mouse neurons, respectively.

The standard extracellular solution contained (in $\mathrm{mM}$ ) $\mathrm{NaCl} \mathrm{140,} \mathrm{KCl}$ 2.4, HEPES 10, glucose 10, $\mathrm{CaCl}_{2} 2$ and $\mathrm{MgCl}_{2} 1$, with $\mathrm{pH} 7.4$ and an adjusted osmotic pressure of 315-320 mOsm. Patch pipettes were filled with an intracellular solution containing (in mM) K-gluconate 146.3, $\mathrm{MgCl}_{2} \mathrm{0.6}$, ATP- $\mathrm{Na}_{2} 4, \mathrm{GTP}-\mathrm{Na}_{2} \mathrm{0}$. phosphocreatine 12, EGTA 1 and HEPES 17.8, as well as creatine phosphokinase $50 \mathrm{U} \mathrm{ml}^{-1}$ ( $\mathrm{pH}$ 7.4). Hypertonic solutions for determining the RRP size were prepared by adding $0.5 \mathrm{M}$ sucrose to the standard extracellular solution. The extracellular solutions were applied using a fast-flow application system (Warner Instruments). Each flow pipe had a diameter of $430 \mu \mathrm{m}$, ensuring that the solution was applied to all parts of an autaptic neuron on the astrocytic microisland $(300 \times 300 \mu \mathrm{m})$. This configuration was necessary to facilitate the application of sucrose to induce the release of RRP vesicles from all nerve terminals of the recorded neuron. All chemicals were purchased from Sigma-Aldrich unless otherwise specified.

DiOlistic labelling. Six- to eight-week-old mice were deeply anaesthetized with $0.1 \mathrm{mg} \mathrm{kg}^{-1}$ phenobarbital and perfused transcardially with $0.1 \mathrm{M}$ phosphate buffer, followed by $50 \mathrm{ml}$ of $2 \%$ paraformaldehyde. The brains were dissected and postfixed in the same fixative for $4 \mathrm{~h}$. Subsequently, sagittal brain sections were obtained using a vibratome and collected in PBS. Twenty-five milligrams of gold particles (Bio-Rad) were coated with $7.0 \mathrm{mg}$ of the lipophilic dye DiI (Invitrogen) and injected into Tefzel tubing, which was subsequently cut into bullets. These particles were delivered DiOlistically at 80 p.s.i. using a Helios gene-gun system (Bio-Rad). The slices were fixed again with $4 \% \mathrm{PFA}$ at $4{ }^{\circ} \mathrm{C}$ for $1 \mathrm{~h}$ and mounted on slides in VectaShield Mounting Medium (Vector Laboratories).

Statistical analysis. Statistical analyses were performed using Student's $t$-test, two-way analysis of variance with proper post hoc tests or the KolmogorovSmirnov test, as specified in each figure legend. Differences were considered significant when $P<0.05$.

\section{References}

1. Fiala, J. C., Spacek, J. \& Harris, K. M. Dendritic spine pathology: cause or consequence of neurological disorders? Brain Res. Brain Res. Rev. 39, 29-54 (2002).

2. Bagni, C. \& Greenough, W. T. From mRNP trafficking to spine dysmorphogenesis: the roots of fragile X syndrome. Nat. Rev. Neurosci. 6, 376-387 (2005).

3. Dindot, S. V., Antalffy, B. A., Bhattacharjee, M. B. \& Beaudet, A. L. The Angelman syndrome ubiquitin ligase localizes to the synapse and nucleus, and maternal deficiency results in abnormal dendritic spine morphology. Hum. Mol. Genet. 17, 111-118 (2008).

4. Niesmann, K. et al. Dendritic spine formation and synaptic function require neurobeachin. Nat Commun. 2, 557 (2011).

5. Crino, P. B., Nathanson, K. L. \& Henske, E. P. The tuberous sclerosis complex. N. Engl. J. Med. 355, 1345-1356 (2006).

6. van Slegtenhorst, M. et al. Identification of the tuberous sclerosis gene TSC1 on chromosome 9q34. Science 277, 805-808 (1997). 
7. Kandt, R. S. et al. Linkage of an important gene locus for tuberous sclerosis to a chromosome 16 marker for polycystic kidney disease. Nat. Genet. 2, 37-41 (1992).

8. Zhang, Y. et al. Rheb is a direct target of the tuberous sclerosis tumour suppressor proteins. Nat. Cell Biol. 5, 578-581 (2003).

9. Inoki, K., Li, Y., Xu, T. \& Guan, K.-L. Rheb GTPase is a direct target of TSC2 GAP activity and regulates mTOR signaling. Genes Dev. 17, 1829-1834 (2003).

10. Zou, J. et al. Rheb1 is required for mTORC1 and myelination in postnatal brain development. Dev. Cell 20, 97-108 (2011).

11. Manning, B. D. \& Cantley, L. C. Rheb fills a GAP between TSC and TOR. Trends Biochem. Sci. 28, 573-576 (2003).

12. Machado-Salas, J. P. Abnormal dendritic patterns and aberrant spine development in Bourneville's disease--a Golgi survey. Clin. Neuropathol. 3, 52-58 (1984).

13. Tavazoie, S. F., Alvarez, V. A., Ridenour, D. A., Kwiatkowski, D. J. \& Sabatini, B. L. Regulation of neuronal morphology and function by the tumor suppressors Tsc1 and Tsc2. Nat. Neurosci. 8, 1727-1734 (2005).

14. Yasuda, S. et al. Activation of Rheb, but not of mTORC1, impairs spine synapse morphogenesis in tuberous sclerosis complex. Sci. Rep. 4, 5155 (2014).

15. Goorden, S. M. I., van Woerden, G. M., van der Weerd, L., Cheadle, J. P. \& Elgersma, Y. Cognitive deficits in Tscl $+/-$ mice in the absence of cerebral lesions and seizures. Ann. Neurol. 62, 648-655 (2007).

16. Hsueh, Y.-P. et al. Direct interaction of CASK/LIN-2 and syndecan heparan sulfate proteoglycan and their overlapping distribution in neuronal synapses. J. Cell Biol. 142, 139-151 (1998).

17. Ethell, I. M. \& Yamaguchi, Y. Cell surface heparan sulfate proteoglycan syndecan-2 induces the maturation of dendritic spines in rat hippocampal neurons. J. Cell Biol. 144, 575-586 (1999).

18. Lin, Y.-L., Lei, Y.-T., Hong, C.-J. \& Hsueh, Y.-P. Syndecan-2 induces filopodia and dendritic spine formation via the neurofibromin-PKA-Ena/VASP pathway. J. Cell Biol. 177, 829-841 (2007).

19. Zimmermann, P. et al. PIP2-PDZ domain binding controls the association of syntenin with the plasma membrane. Mol. Cell 9, 1215-1225 (2002).

20. Hirbec, H., Martin, S. \& Henley, J. M. Syntenin is involved in the developmental regulation of neuronal membrane architecture. Mol. Cell. Neurosci. 28, 737-746 (2005).

21. Long, X., Lin, Y., Ortiz-Vega, S., Busch, S. \& Avruch, J. The Rheb switch 2 segment is critical for signaling to target of rapamycin complex 1. J. Biol. Chem. 282, 18542-18551 (2007).

22. Yeung, R. S. et al. Predisposition to renal carcinoma in the Eker rat is determined by germ-line mutation of the tuberous sclerosis 2 (TSC2) gene. Proc. Natl Acad. Sci. USA 91, 11413-11416 (1994).

23. Hamilton, A. M. et al. Activity-dependent growth of new dendritic spines is regulated by the proteasome. Neuron 74, 1023-1030 (2012).

24. Hung, A. Y., Sung, C. C., Brito, I. L. \& Sheng, M. Degradation of postsynaptic scaffold GKAP and regulation of dendritic spine morphology by the TRIM3 ubiquitin ligase in rat hippocampal neurons. PLoS ONE 5, e9842 (2010).

25. Beekman, J. M. \& Coffer, P. J. The ins and outs of syntenin, a multifunctional intracellular adaptor protein. J. Cell. Sci. 121, 1349-1355 (2008).

26. Ethell, I. M. et al. EphB/Syndecan-2 signaling in dendritic spine morphogenesis. Neuron 31, 1001-1013 (2001).

27. Chao, H.-W., Hong, C.-J., Huang, T.-N., Lin, Y.-L. \& Hsueh, Y.-P. SUMOylation of the MAGUK protein CASK regulates dendritic spinogenesis. J. Cell Biol. 182, 141-155 (2008).

28. Biederer, T. \& Südhof, T. C. CASK and protein 4.1 support F-actin nucleation on neurexins. J. Biol. Chem. 276, 47869-47876 (2001).

29. Cohen, A. R. et al. Human CASK/LIN-2 binds syndecan-2 and protein 4.1 and localizes to the basolateral membrane of epithelial cells. J. Cell Biol. 142, 129-138 (1998).

30. Xu, N.-J., Sun, S., Gibson, J. R. \& Henkemeyer, M. A dual shaping mechanism for postsynaptic ephrin-B3 as a receptor that sculpts dendrites and synapses. Nat. Neurosci. 14, 1421-1429 (2011).

31. McClelland, A. C., Hruska, M., Coenen, A. J., Henkemeyer, M. \& Dalva, M. B. Trans-synaptic EphB2-ephrin-B3 interaction regulates excitatory synapse density by inhibition of postsynaptic MAPK signaling. Proc. Natl Acad. Sci. USA 107, 8830-8835 (2010).

32. Aoto, J. et al. Postsynaptic ephrinb3 promotes shaft glutamatergic synapse formation. J. Neurosci. 27, 7508-7519 (2007).

33. Alonso, M., Medina, J. H. \& Pozzo-Miller, L. ERK1/2 activation is necessary for BDNF to increase dendritic spine density in hippocampal CA1 pyramidal neurons. Learn. Mem. 11, 172-178 (2004).

34. McClelland, A. C., Sheffler-Collins, S. I., Kayser, M. S. \& Dalva, M. B. Ephrin-B1 and ephrin-B2 mediate EphB-dependent presynaptic development via syntenin-1. Proc. Natl Acad. Sci. USA 106, 20487-20492 (2009).

35. Casanova, E. et al. A CamKII $\alpha$ iCre BAC allows brain-specific gene inactivation. Genesis 31, 37-42 (2001).

36. Kobayashi, T. et al. Renal carcinogenesis, hepatic hemangiomatosis, and embryonic lethality caused by a germ-line Tsc2 mutation in mice. Cancer Res. 59, 1206-1211 (1999).
37. Bai, X. et al. Rheb activates mTOR by antagonizing its endogenous inhibitor, FKBP38. Science 318, 977-980 (2007).

38. Sun, Y. et al. Phospholipase D1 is an effector of Rheb in the mTOR pathway Proc. Natl Acad. Sci. USA 105, 8286-8291 (2008).

39. Meikle, L. et al. Response of a neuronal model of tuberous sclerosis to mammalian target of rapamycin (mTOR) inhibitors: effects on mTORC1 and Akt signaling lead to improved survival and function. J. Neurosci. 28, 5422-5432 (2008).

40. Lipinski, M. M. et al. A Genome-wide siRNA screen reveals multiple mTORC1 independent signaling pathways regulating autophagy under normal nutritional conditions. Dev. Cell. 18, 1041-1052 (2010).

41. Neuman, N. A. \& Henske, E. P. Non-canonical functions of the tuberous sclerosis complex-Rheb signalling axis. EMBO Mol. Med. 3, 189-200 (2011).

42. Carson, R. P., Van Nielen, D. L., Winzenburger, P. A. \& Ess, K. C. Neuronal and glia abnormalities in Tsc1-deficient forebrain and partial rescue by rapamycin. Neurobiol. Dis. 45, 369-380 (2012).

43. Okabe, S., Miwa, A. \& Okado, H. Spine formation and correlated assembly of presynaptic and postsynaptic molecules. J. Neurosci. 21, 6105-6114 (2001).

44. Biederer, T. et al. SynCAM, a synaptic adhesion molecule that drives synapse assembly. Science 297, 1525-1531 (2002).

45. Koroll, M., Rathjen, F. G. \& Volkmer, H. The neural cell recognition molecule neurofascin interacts with syntenin-1 but not with syntenin-2, both of which reveal self-associating activity. J. Biol. Chem. 276, 10646-10654 (2001).

46. Enz, R. \& Croci, C. Different binding motifs in metabotropic glutamate receptor type $7 \mathrm{~b}$ for filamin $\mathrm{A}$, protein phosphatase $1 \mathrm{C}$, protein interacting with protein kinase C (PICK) 1 and syntenin allow the formation of multimeric protein complexes. Biochem. J. 372, 183-191 (2003).

47. Saitsu, H. et al. CASK aberrations in male patients with Ohtahara syndrome and cerebellar hypoplasia. Epilepsia 53, 1441-1449 (2012).

48. Ohtahara, S. \& Yamatogi, Y. Epileptic encephalopathies in early infancy with suppression-burst. J. Clin. Neurophysiol. 20, 398-407 (2003).

49. Grootjans, J. J., Reekmans, G., Ceulemans, H. \& David, G. Syntenin-syndecan binding requires syndecan-synteny and the co-operation of both PDZ domains of syntenin. J. Biol. Chem. 275, 19933-19941 (2000).

50. Hata, Y., Butz, S. \& Sudhof, T. CASK: a novel dlg/PSD95 homolog with an $\mathrm{N}$-terminal calmodulin-dependent protein kinase domain identified by interaction with neurexins. J. Neurosci. 16, 2488-2494 (1996).

51. Meikle, L. et al. A mouse model of tuberous sclerosis: neuronal loss of Tsc1 causes dysplastic and ectopic neurons, reduced myelination, seizure activity, and limited survival. J. Neurosci. 27, 5546-5558 (2007).

52. Muncy, J., Butler, I. J. \& Koenig, M. K. Rapamycin reduces seizure frequency in tuberous sclerosis complex. J. Child Neurol. 24, 477 (2009).

53. Zeng, L. H., Rensing, N. R. \& Wong, M. The mammalian target of rapamycin signaling pathway mediates epileptogenesis in a model of temporal lobe epilepsy. J. Neurosci. 29, 6964-6972 (2009).

54. Zeng, L. H., Xu, L., Gutmann, D. H. \& Wong, M. Rapamycin prevents epilepsy in a mouse model of tuberous sclerosis complex. Ann. Neurol. 63, 444-453 (2008).

55. Krueger, D. A. et al. Everolimus for subependymal giant-cell astrocytomas in tuberous sclerosis. N. Engl. J. Med. 363, 1801-1811 (2010).

56. Franz, D. N. et al. Efficacy and safety of everolimus for subependymal giant cell astrocytomas associated with tuberous sclerosis complex (EXIST-1): a multicentre, randomised, placebo-controlled phase 3 trial. Lancet 381, 125-132 (2013).

57. Krueger, D. A. et al. Everolimus treatment of refractory epilepsy in tuberous sclerosis complex. Ann. Neurol. 74, 679-687 (2013).

58. Yamagata, K. et al. rheb, a growth factor- and synaptic activity-regulated gene, encodes a novel Ras-related protein. J. Biol. Chem. 269, 16333-16339 (1994).

59. Rennebeck, G. et al. Loss of function of the tuberous sclerosis 2 tumor suppressor gene results in embryonic lethality characterized by disrupted neuroepithelial growth and development. Proc. Natl Acad. Sci. USA 95, 15629-15634 (1998).

60. Chevray, P. M. \& Nathans, D. Protein interaction cloning in yeast: identification of mammalian proteins that react with the leucine zipper of Jun. Proc. Natl Acad. Sci. USA 89, 5789-5793 (1992).

61. Irie, Y. et al. Molecular cloning and characterization of Amida, a novel protein which interacts with a neuron-specific immediate early gene product Arc, contains novel nuclear localization signals, and causes cell death in cultured cells. J. Biol. Chem. 275, 2647-2653 (2000).

62. Long, X., Lin, Y., Ortiz-Vega, S., Yonezawa, K. \& Avruch, J. Rheb binds and regulates the mTOR kinase. Curr. Biol. 15, 702-713 (2005).

63. Sugiura, H. et al. Inhibitory role of endophilin 3 in receptor-mediated endocytosis. J. Biol. Chem. 279, 23343-23348 (2004).

64. Yasuda, S. et al. Activity-induced protocadherin Arcadlin regulates dendritic spine number by triggering $\mathrm{N}$-cadherin endocytosis via TAO2[beta] and p38 MAP kinases. Neuron 56, 456-471 (2007). 
65. Wojcik, S. M. et al. A shared vesicular carrier allows synaptic corelease of GABA and glycine. Neuron 50, 575-587 (2006).

66. Pyott, S. J. \& Rosenmund, C. The effects of temperature on vesicular supply and release in autaptic cultures of rat and mouse hippocampal neurons. J. Physiol. 539, 523-535 (2002).

67. Burgalossi, A. et al. Analysis of neurotransmitter release mechanisms by photolysis of caged $\mathrm{Ca} 2+$ in an autaptic neuron culture system. Nat. Protoc. 7, 1351-1365 (2012)

\section{Acknowledgements}

We are grateful to Y. Matsumoto and K. Kohyama for the gene gun, T. Shimada for technical assistance, A. Sato and K. Ikeda for $T s c 2^{+/-}$mice, and H. Tanaka for critical reading of the manuscript. This work was supported by JSPS KAKENHI grant number 24659093 and 25293239, MEXT KAKENHI grant number 23110525 and 25110737, and a grant from the Naito Foundation.

\section{Author contributions}

K.Y., H.S., S.Y. and S.K. designed the research; H.S., S.Y., S.K., H. K., K.E., K.T., K.I., M.I. T.K., O.H. and K.Y. performed the experiments; and K.Y., H.S., S.Y. and S.K. wrote the manuscript. All authors reviewed the manuscript.

\section{Additional information}

Supplementary Information accompanies this paper at http://www.nature.com/ naturecommunications

Competing financial interests: The authors declare no competing financial interests.

Reprints and permission information is available online at http://npg.nature.com/ reprintsandpermissions/

How to cite this article: Sugiura, H. et al. Rheb activation disrupts spine synapse formation through accumulation of syntenin in tuberous sclerosis complex. Nat. Commun. 6:6842 doi: 10.1038/ncomms7842 (2015). 